\title{
Status Report to the ERDA Nuclear Data Committee
}

\author{
F. G. Perey \\ J. C. Gentry
}

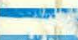




\section{DISCLAIMER}

This report was prepared as an account of work sponsored by an agency of the United States Government. Neither the United States Government nor any agency Thereof, nor any of their employees, makes any warranty, express or implied, or assumes any legal liability or responsibility for the accuracy, completeness, or usefulness of any information, apparatus, product, or process disclosed, or represents that its use would not infringe privately owned rights. Reference herein to any specific commercial product, process, or service by trade name, trademark, manufacturer, or otherwise does not necessarily constitute or imply its endorsement, recommendation, or favoring by the United States Government or any agency thereof. The views and opinions of authors expressed herein do not necessarily state or reflect those of the United States Government or any agency thereof. 


\section{DISCLAIMER}

Portions of this document may be illegible in electronic image products. Images are produced from the best available original document. 
Printed in the United States of America. Available from

National Technical Information Service

U.S. Department of Commerce

5285 Port Royal Road, Springfield, Virginia 22161

Prıce: Prınted Lopy $\$ 4.500$; Microfiche $\$ 2.25$

This report was prepared as an account of work sponsored by the United States Government. Neither the United States nor the Energy Research and Development Administration/United States Nuclear Regulatory Commission, nor any of their employees, nor any of their contractors, subcontractors, or their employees, makes any warranty, express or implied, or assumes any legal liability or responsibility for the accuracy, completeness or usefulness of any information, apparatus, product or process disclosed, or represents that its use would not infringe privately owned rights. 
Contract No. W-7405-eng-26

Neutron Physics Division

STATUS REPORT TO THE ERDA NUCLEAR DATA COMMITTEE

F. G. Perey and J. C. Gentry

MAY 1976

NOTICE This document contains information of a preliminary nature and was prepared primarily for internal use at the Oak Ridge National Laboratory. It is subject to revision or correction and thereforo does not ropresent a final report.

OAK RIDGE NATIONAL LABORATORY

Oak Ridge, Tennessee 37830

operated by

UNION CARBIDE CORPORATION

for the

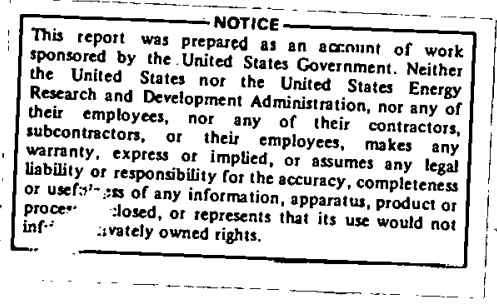

ENERGY RESEARCH AND DEVELOPMENT ADMINISTRATION 
THIS PAGE

\section{WAS INTENTIONALLY LEFT BLANK}


CONTENTS

Page

ABSTRACT . . . . . . . . . . . . . . . . . . v

CROSS SECTION MEASUREMENTS . . . . . . . . . . . . . . . 1

Gamma-Ray Production Data . . . . . . . . . . . . 1

Capture Cross Sections . . . . . . . . . . . . . 4

Total Cross Sections . . . . . . . . . . . . 11

Scattering and Reactions .............. 14

Capture $\gamma$-Rays ................ . 17

Actinides .................. . 20

Integral Measurements . . . . . . . . . . . 38

Experimental Techniques ............... 40

DATA ANALYSES ................................. 41

Theoretical Calculations . . . . . . . . . . 4 41

ENDF/B Related Evaluations . . . . . . . . . . 42

Validation of ENDF/B Evaluations

Through Integral Measurements . . . . . . . . . . . 44

Sensitivity Studies . . . . . . . . . . . . . . 46

Multigroup Libraries . . . . . . . . . . . . . 49

COMPILATION AND NUCLEAR DATA PROJECT . . . . . . . . . . 52

Compilation of Phenomenological

Optical-Model Parameters ............ 52

Nuclear Data Project Activities - 1975 . . . . . . . 52 
THIS PAGE

WAS INTENTIONALLY

LEFT BLANK 


\section{ABSTRACT}

This report was prepared for the ERDA-NDC and covers work performed at ORNL since May 1975 in areas of nuclear data of relevance to the U.S. applied nuclear energy program. The report was mostly generated through a review of abstracts of work completed to the point of being subjected to some form of publication in the open literature, formal ORNL reports, ORNL technical memoranda, progress reports, or being presented at technical conferences. As much as possible we have reproduced the complete abstract of the original publication with only minor editing. In a few cases progress reports were written specifically for this publication. The authors have selected the materials to be included in this report on the basis of perceived interests of ERDA-NDC members and cannot claim completeness. 


\section{A. CROSS SECTION MEASUREMENTS}

\section{Gamma-Ray Production Data}

During the reporting period the following gamma-ray production data obtained at ORELA were released:

a. Production of Low Energy Gamma Rays by Neutron Interactions with Fluorine for Incident Neutron Energies Between 0.1 and $20 \mathrm{MeV}^{\star}, \star \star$ (G. L. Morgan and J. K. Dickens)

Differential cross sections for the production of low-energy gamma rays (<240 keV) by neutron interactions in fluorine have been measured for neutron energies between 0.1 and $20 \mathrm{MeV}$. The Oak Ridge Electron Linear Accelerator was used as the neutron source. Gamma rays were detected at $92^{\circ}$ using an intrinsic germanium detector. Incident neutron energies were determined by time-of-flight techniques. Tables are presented for the production cross sections of three gamma rays having energies of 96,110 , and $197 \mathrm{keV}$.

b. The $V(n, x y)$ Reaction Cross Section for Incident Neutron Energies Between 0.2 and $20.0 \mathrm{MeV}+\neq$ (E. Newman and G. L. Morgan)

Differential cross sections for the neutron-induced gamma-ray production from natural vanadjum have been measured for incident neutron energies between 0.2 and $20.0 \mathrm{MeV}$. The Oak Ridge Electron Linear Accelerator (ORELA) was used to provide the neutrons and a $\mathrm{NaI}$ spectrometer to detect the gamma rays at $125^{\circ}$. The data presented are the double differential cross section, $d^{2} \sigma / d \Omega d E$, for gamma-ray energies between 0.3 and $10.6 \mathrm{MeV}$ for coarse intervais in incident neutron energy. The integrated yield of gamma rays of energies greater than $300 \mathrm{keV}$ and higher resolution in the neutron energy is also presented. The experimental results are compared with the Evaluated Neutron Data Files (ENDF).

* Abstract of ORNL-TM-4823.

** Relevant to request No. 74180 .

+ Abstract of ORNL-TM-5299, ENDF-221.

$\neq$ Relevant to request No. 74224 . 
c. The $\operatorname{Cr}(n, x y)$ Reaction Cross Section for Incident Neutron Energies Between 0.2 and $20.0 \mathrm{MeV}^{\star}, \star \star \quad$ (G. L. Morgan and E. Newman)

Differential cross sections for the neutron-induced gamma-ray production from natural chromium have been measured for incident neutron energies between 0.2 and $20.0 \mathrm{MeV}$. The Oak Ridge Electron Linear Accelerator (ORELA) was used to provide the neutrons and a NaI spectrometer to detect the gamma rays at the laboratory angle of $125^{\circ}$. The data presented are the double differential cross section, $d^{2} \sigma / d \Omega d E$, for gamma-ray energies between 0.3 and $10.6 \mathrm{MeV}$ for coarse intervals in incident neutron energy. The integrated yield of gamma rays of energies greater than 300 $\mathrm{keV}$ and higher resolution in the neutron energy is also presented. The experimental results are compared with the Evaluated Neulrun Data Flles (ENDF).

d. The $\mathrm{Cu}(n, x y)$ Reaction Cross Section for Incident Neutron Energies Between 0.2 and $20.0 \mathrm{MeV}+, \neq$ (G. T. Chapman)

Differential cross sections for the neutron-induced gamma-ray production from copper have been measured for incident neutron energies between 0.2 and 20.0 MeV. The nak Ridge Electron Lincar Acceleralur (URELA) was used to provide the neutrons and a NaI spectrometer to detect the gamma rays at $125^{\circ}$. The data presented are the doubly differential cross section, $\mathrm{d}^{2} \sigma / \mathrm{d} \Omega \mathrm{dE}$, for gamma-ray energies between 0.3 and $10.6 \mathrm{MeV}$ for coarse intervals in incident neutron energy. The integrated yield of gamma rays of energies greater than $300 \mathrm{keV}$ with higher resolution in the neutron energy is also presented. The experimental results are compared with previous measurements made at ORELA and with the Evaluated Neutron Data File (ENDF/B-IV, MAT 1295).

e. The $\mathrm{Nb}(n, x y)$ Reaction Cross Section for Incident Neutron Energies Between 0.65 and $20.0 \mathrm{MeV}$ " (Dickens, Morgan and Newman)

Differential cross sections for the neutron-induced gamma-ray production from niobium have been measured for neutron energies between 0.65 and $20 \mathrm{MeV}$. The Oak Ridge Electron Linear Accelerator (ORELA) was used to provide the neutrons and a $\mathrm{NaI}$ spectrometer to detect the gamma rays at $90^{\circ}$. The data presented are the double differential cross section, $d^{2} \sigma / d \Omega d E$, for gamma-ray energies between 0.75 and $10.5 \mathrm{MeV}$ and coarse intervals in the incident neutron energy. The integrated yield of gamma rays of energies greater than $0.75 \mathrm{MeV}$ and higher resolution

* Abstract of ORNL-TM-5098, ENDF-222.

** Relevant to request Nos. 74230 and 72037 .

+ Abstract of ORNL-TM-5215.

₹ Relevant to request No. 74304.

I Abstract of ORNI.-TM-4972, ENDF-219. 
in the incident neutron energy is also presented. The experimentally determined results are compared with the Evaluated Neutron Data Library (ENDL).

f. The $M o(n, x y)$ Reaction Cross Section for Incident Neutron Energies Between 0.2 and 20.0 MeV*,** (G. L. Morgan and E. Newman)

Differential cross sections for the neutron-induced gamma-ray production from natural molybdenum have been measured for incident neutron energies between 0.2 and $20.0 \mathrm{MeV}$. The Oak Ridge Electron Linear Accelerator (ORELA) was used to provide the neutrons and a $\mathrm{NaI}$ spectrometer to detect the gamma rays at $125^{\circ}$. The data presented are the double differential cross section, $d^{2} \sigma / d \Omega d E$, for gamma-ray energies between 0.3 and $10.6 \mathrm{MeV}$ for coarse intervals in incident neutron energy. The integrated yield of gamma rays of energies greater than $300 \mathrm{keV}$ and higher resolution in the neutron energy is also presented. The experimental results are compared with the Evaluated Neutron Data Files (ENDF).

g. Gamma-Ray Production due to Neutron Interactions with Silver for Incident Neutron Energies Between 0.3 and $20 \mathrm{MeV}$ : Tabulated Differential Cross Sectionst (Dickens, Love and Morgan)

Numerical values of differential cross sections for gamma rays produced by neutron reactions with silver have been obtained for neutron energies between 0.3 and $20 \mathrm{MeV}$ for $\theta=125^{\circ}$. The $\mathrm{d}^{2} \sigma / \mathrm{d} \omega \mathrm{dE}$ values were obtained using a $\mathrm{NaI}$ spectrometer. These data are presented as gamma-ray production group cross-section values of $\mathrm{d}^{2} \sigma / d \omega d \mathrm{~d}$ for $0.3<E<10.5 \mathrm{MeV}$, with gamma-ray intervals ranging from $15 \mathrm{keV}$ for $\mathrm{E}_{\mathrm{s}}<0.4 \overline{\mathrm{MeV}}^{\gamma}$ t $\overline{0} 160 \mathrm{keV}$ for $E_{\gamma} \sim 9 \mathrm{MeV}$. Neutron energy intervals varied from $0.10 \mathrm{MeV}$ for $E_{n}$ between 0.3 and $0.4 \mathrm{MeV}$ to $3 \mathrm{MeV}$ for $E_{n}$ between 14 and $20 \mathrm{MeV}$.

h. The $A u(n, x y)$ Reaction Cross Section for Incident Neutron Energies Between 0.2 and $20.0 \mathrm{MeV} \neq$ (G. L. Morgan and E. Newman)

Differential cross sections for the neutron-induced gamma-ray production from natural gold have been measured for incident neutron energies between 0.2 and $20.0 \mathrm{MeV}$. The Oak Ridge Electron Linear Accelerator (ORELA) was used to provide the neutrons and a NaI spectrometer to detect the gamma rays at $125^{\circ}$. The data presented are the double differential cross sections, $d^{2} \sigma / d \Omega d E$, for gamma-ray energies between 0.3 and $10.6 \mathrm{MeV}$ for coarse intervals in incident neutron energy. The integrated yield of gamma rays of energies greater than $300 \mathrm{keV}$ and higher resolution in the neutron energy is also presented. The experimental results are compared with the Evaluated Neutron Data Library (ENDL).

* Abstract of ORNL-TM-5097, ENDF-220.

** Relevant to request No. 74313 .

+ Abstract of ORNL-TM-5081.

$\neq$ Abstract of ORNL-TM-4973. 


\section{Capture Cross Sections}

The following are abstracts of papers written or published during the reporting period:

a. Neutron Capture and Transmission by ${ }^{24,25,26} \mathrm{Mg} *, \star \star$ (H. Weigmann, + R. L. Macklin and J. A. Harvey)

Resonance neutron capture and transmission by the stable isotopes of magnesium were measured at the Oak Ridge Electron Linear Accelerator time-of-flight facility; capture by separated isotope and natural metal samples at 40 meters and transmission by a natural metal sample $(78.7 \%$ ${ }^{24} \mathrm{Mg}$ ) at 200 meters. Twerily-six resonances in ${ }^{24} \mathrm{Mg}+\mathrm{n}$ up to $1.8 \mathrm{MeV}$ were fitted with R-matrix parameters. The data werc sufficient to assicin spin and parity to 19 ur lhese. The capture data were analyzed up to $850 \mathrm{keV}$ for ${ }^{24} \mathrm{MeV}+\mathrm{n}, 265 \mathrm{keV}$ for ${ }^{25} \mathrm{Mg}+\mathrm{n}\left(17^{+}\right.$resonances), and $440 \mathrm{keV}$ for ${ }^{26} \mathrm{Mg}+n$ (4 resnnances). Average capturc at stellar interinr permer alures was calculated. The ${ }^{24} \mathrm{Mg}+\mathrm{n}$ data serve to assess the isospin impurities in three isobaric analogue states. Three other states exhibit reduced neutron widths each several percent of the Wigner limit which may be understood in terms of simple shell model configurations.

b. The Neutron Capture Cross Section of Natural Silicont, $\pi$ (Boldeman, $\S$ Allen, $\S$ Musgrove $\S$ and Macklin)

The neutron capture cross section of natural silicon has been measured to $1500 \mathrm{keV}$ using the capture cross section facility at the $40 \mathrm{~m}$ station of the Oak Ridge Electron Linear Accelerator. Analys is of the present data, in combination with existing total cross section information, has provided almost complete resonance data for ${ }^{28} \mathrm{Si}$. On the other hand, the capture kernel only $\left(\mathrm{g} \Gamma \Gamma_{n} \Gamma_{\gamma} / \Gamma\right)$ has heen obtained for ncutron capture resonances in ${ }^{2 y} \mathrm{Si}$ and ${ }^{30} \mathrm{Si}$. A st.rong positive corrclation has been observed between the radiative width and the corresponding reduced neutron width for $p$-wave resonances in ${ }^{28} \mathrm{Si}$, confirming significant valence effects. It is noted that a quantitative valence calculation provides only an approximate estimate of the valence strength for this nucleus. It was not possible to confirm from the present measurements, reported asymmetry observed in two resonances in the ${ }^{29} \mathrm{Si}(\gamma, n)$ reaction.

* To be published in Phys. Rev. C.

** Relevant to request No. 74181 .

+ Visiting Srientist frün CBNM, Geel, Belgium.

$\neq$ Nuclear Physics A252, 62 (1975).

T Relevant to request No. 74182.

5 Australian Atomic Energy Commission, Lucas Heights, NSW, Australia. 
c. Kilovolt ${ }^{3}{ }^{3} S\left(n, \alpha_{0}\right)$ and ${ }^{33} S(n, \gamma)$ Cross Sections: Importance in

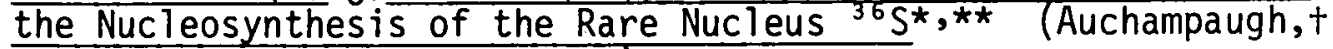
Halperin, Macklin and Howardf)

The ${ }^{3} S\left(n, \alpha_{0}\right)$ and ${ }^{3} 3(n, \gamma)$ cross sections have been measured from $\sim 10$ to $\sim 700 \mathrm{keV}$. Resonance parameters are given for 39 resonances. The level spacing is determined to be $9.1 \pm 0.9 \mathrm{keV}$. The $\sigma\left(n, \alpha_{0}\right)$ and $\sigma(n, \gamma)$ cross sections are averaged over a Maxwellian distribution for values of kT from 25 to $275 \mathrm{keV}$. When these cross sections are used in a nucleosynthesis calculation of the rare isotope ${ }^{3.6} \mathrm{~S}$, the overproduction of this isotope, relative to the other nuclei formed in the universe, is reduced from a factor of 10 to 2.5.

d. Resonant Neutron Capture in ${ }^{40} \mathrm{Ca} \pi, \S$ (Musgrove, Allen, Boldeman, Chan, ${ }^{+}$and Mackl in)

The neutron capture cross section of ${ }^{40} \mathrm{Ca}$ has been measured with $\sim 0.2$ percent energy resolution below $E_{n}=300 \mathrm{keV}$. Resonance parameters have been extracted for many new $p$ - and $d$-wave resonances.

Gamma-ray spectra were also measured following capture in one doublet and two resolved resonances below $50 \mathrm{keV}$. Strong feeding of low lying p-wave levels was observed in all. cases. Calculations showed that valence transitions were inadequate to account for the observed dominance of these transitions and a further mechanism is required.

The average resonance parameters obtained from the data are as follows: $\langle D\rangle=37 \pm 4 \mathrm{keV}, 10^{4} \mathrm{~S}_{1}=0.16 \pm 0.05,10^{4} \mathrm{~S}_{1}=.2 .0 \pm 0.7$. The average radiative widths and standard deviations of their distributions were found to be strongly l-dependent as follows: $\left\langle\Gamma_{\gamma}\right\rangle_{s}=1.5 \pm 0.9 \mathrm{eV}$, $\left\langle\Gamma_{\gamma}\right\rangle_{p}=0.36 \pm 0.09 \mathrm{eV}$ and $\left\langle\Gamma_{\gamma}\right\rangle_{d}=0.75 \pm 0.36 \mathrm{eV}$.

* Phys. Rev. C 12, 1126 (1975).

** Relevant to request Nos. 74023 and 74024 .

+ Los Alanlus Scienlific Laboratory, Los Alamos, New Mexico 87544.

\# Astronomy Department, University of Illinois, Urbana, I11inois 61801 .

I Submitted for publication in Nuclear Physics.

$\S$ Relevant to request Nos. 74029 and 74030 .

+ University of Melbourne, Victoria, Australia. 
e. Neutron Capture Cross Section of ${ }^{59} \mathrm{Co}$ in the Energy Range

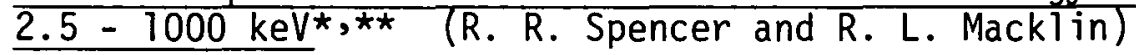

Time-of-flight measurements of the capture cross section of ${ }^{59} \mathrm{Co}$ were carried out in the neutron energy region 2.5 - $1000 \mathrm{keV}$ using the Oak Ridge Electron Linear Accelerator and a pair of nonhydrogenous liquidscintillator gamma-ray detectors. Resonance energies and capture areas were determined for a large number of resolved resonances up to $85 \mathrm{keV}$ neutron energy, and radiation widths for 35 known s-wave resonances were derived. Significant positive correlations $(\rho \simeq 0.3)$ were found between the radiative widths and neutron reduced widths of these s-wave resonances for both possible spin states.

f. High Resolution Neutron Capture Cross Sections in ${ }^{63} \mathrm{Cu}$ and ${ }^{65} \mathrm{Cu}+, \neq$ (M. S. Pandey, I J. B. Larg, fl R. Macklin and J. Häperin)

Neutron capture cross sections in separated isotopes of copper $\left({ }^{63} \mathrm{Cu}\right.$ and $\left.{ }^{65} \mathrm{Cu}\right)$ from a few keV to a few hundreds of keV. were measured using the ORELA time-of-flight facility. From these measurements the capture yields of resonances were accurately determined giving precise values of $\mathrm{g} \Gamma_{\mathrm{n}}$ and $\Gamma_{\gamma}$ up to a maximum neutron energy of $50 \mathrm{keV}$. For ${ }^{63} \mathrm{Cu}$ one obtains a value of $\left\langle\Gamma_{\gamma}\right\rangle=(507 \pm 30) \mathrm{meV}, \mathrm{p}$-wave average level spacing $\langle D\rangle_{l=1}=(.495 \pm .026) \mathrm{keV}$ and strength function $\left.S_{1}=(0.44 \pm 0.07) 10^{-4} \mathrm{eV}^{-1}\right)_{2}$. The corresponding $s$-wave mean level spacings for ${ }^{63} \mathrm{Cu}$ and ${ }^{65} \mathrm{Cu}$ are $(1.04 \pm 0.11) \mathrm{keV}$ and $(1.47 \pm 0.19) \mathrm{keV}$ respectively. Some few of the narrowest levels have been assumed formed by $d$-waves. g. Valence Neutron Capture in ${ }^{88} \mathrm{Sr} \S$ (Boldeman, Allen, Musgrove and

The neutron capture cross section of ${ }^{88} \mathrm{Sr}$ has been measured with high energy resolution between 2.5 and $400 \mathrm{keV}$ using the capture cross section facility at the $40 \mathrm{~m}$ station on the Oak Ridge Electron Linear Accelerator. Strong positive correlations have been observed between the reduced neutron and radiative widths for both $p_{3 / 2}$ and $p_{1 / 2}$ resonances. The data provide an unambiguous quantitative verification of the valence model.

* Submitted for publication in Nucl. Sci. Eng.

** Relevant to request No. 69106 .

t Submitted for publication in Phys. Rev.

$\neq$ Relevant to request Nos. 6913? and 74307 .

I State University of New York at Albany.

$\S$ Submitted for publication in Nuclear Physics. 
h. Valence Component in the Neutron Capture Cross Section of ${ }^{90} \mathrm{Zr} *, * *$

The neutron capture cross section of $90 \mathrm{Zr}$ has been measured with high energy resolution between 3 and $200 \mathrm{keV}$ using the capture cross section facility at the $40 \mathrm{~m}$ station on the Oak Ridge Electron Linear Accelerator. Through the comparison of the present data with the total cross-section and inverse ${ }^{91} \mathrm{Zr}(\gamma, n)$ data from Toohey and Jackson, complete resonance parameters have been extracted for $37 p_{3}, 2$ and $p_{1 / 2}$ and 11 swave resonances out of a total of 101 observed resonances. The neutron strength functions extracted from the resonance parameters are $S_{0}=$ $0.56 \times 10^{-4}, S_{1}=3.8 \times 10^{-4}, S_{1}\left(p_{3} / 2\right)=4.7 \times 10^{-4}$ and $S_{1}\left(p_{1 / 2}\right)=1.9 \times$ $10^{-4}$. It is noteworthy that the $S_{1}\left(p_{3 / 2}\right)$ strength function is significantly larger than the $S_{1}\left(p_{1 / 2}\right)$ strength function in agreement with theoretical expectation.

A significant correlation $(\rho=0.58)$ exists between the reduced neutron widths and the radiative widths for the $37 p_{3 / 2}$ resonances. The data give strong confirmation of the valence neutron model. With standard valence calculations all radiative widths can be calculated reasonably from the associated reduced neutron width. However, to explain the measured correlation coefficient, it has been necessary to include with the valence component, single-particle transitions to the ground and low excited states of ${ }^{91} \mathrm{Zr}$, which are uncorrelated with the resonance reduced neutron width.

The average capture $\gamma$-spectrum for neutron capture in ${ }^{90} \mathrm{Zr}$ between 2 and $80 \mathrm{keV}$ has been calculated from the valence model and the present data and is found to be in very close agreement with published experimental data.

i. Neutron Capture Cross Section of Niobium-93 from 2.6 to $700 \mathrm{keV}+$, $\neq$ TR. L. Macklin)

The neutron capture cross section of stable ${ }^{93} \mathrm{Nb}$ was measured by time-of-flight methodology at the Oak Ridge Electron Linear Accelerator. Individual resonances were parameterized to $7.4 \mathrm{keV}$ with energy resolution $\leq 0.14 \%$ full-width-at-half-maximum. The average cross section was deduced from 3 to $700 \mathrm{keV}$ with an accuracy estimated at 3 to $5 \% \mathrm{SD}$. The average data to $100 \mathrm{keV}$ are well fitted by strength functions, but the fluctuations about the fit are not consistent with an energy-independent level density proportional to $2.1+1$ beyond $20 \mathrm{keV}$.

* Nuclear Physics A246, 1 (1975).

** Relevant to request Nos. 69142,69151 and 72062 .

+ Nucl. Sci. Eng. 59, 12 (1976).

$\neq$ Relevant to request Nos. 62049 and 62050. 
j. Average Neutron Resonance Parameters and Radiative Capture Mechanisms for the Isotopes of Molybdenum*, $\star *$

(Musgrove, Allen, Boldeman and Macklin)

The neutron capture cross sections of the stable molybdenum isotopes have been measured with high energy resolution between 3 and $90 \mathrm{keV}$ at the $40 \mathrm{~m}$ station of ORELA. Average resonance parameters are extracted for $s$ - and $p$-wave resonances. The $s$-wave neutron strength function is close to $0: 5 \times 10^{-4}$ for all isotopes, but the $p$-wave strength function exhibits a well defined peak near $A \sim 95$.

Both s- and $p$-wave radiative widths decrease markedly as further neutrons are added to the closed shell. The $p$-wave radiative widthis are generally greater than the s-wave widths showing the presence of nonstatistical i-decay mechanisms.

Valence neutron theory fails to explain the magnitude of the $p$ to s-wave radiative width discrepancy and doorway state processes are invoked. The existing data for ${ }^{92} \mathrm{Mo}$ and ${ }^{9 \mathrm{~B}} \mathrm{Mo}$ are reviewed and the possible radiative mechanisms are outlined.

In particular, the data for ${ }^{98}$ Mo provide a clear violation of the usual valence theory, since no correlations between radiative and neutron strengths are found. Further, the radiative widths are far smaller than can be explained on the valence model. An explanation for this loss of valence strength is advanced.

Interpolated resonance parameters allow an estimate for the unknown cross section for ${ }^{9} \mathrm{Mo}(n, \gamma)$.

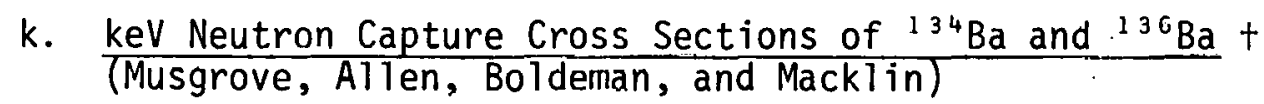

The neutron capture cross sections of ${ }^{13{ }^{4}} \mathrm{Ba}$ and ${ }^{136} \mathrm{Ba}$ have been measured in the energy region 3 to $100 \mathrm{keV}$. The following average quantities were deduced from the extracted resonance parameters: $\angle D\rangle=$ $127 \pm 10 \mathrm{eV}, 10^{4} \mathrm{~S}_{0}=0.85 \pm 0.3,10^{4} \mathrm{~S}_{1}=0.8,\left\langle\Gamma \gamma^{>}=120 \pm 20 \mathrm{MeV}\right.$ for ${ }^{13}{ }^{4} \mathrm{Ba}$. Analysis of the ${ }^{136} \mathrm{Ba}$ data gave $\left\langle\Gamma_{\gamma}\right\rangle=125 \pm 30 \mathrm{meV}$ for $s$-wave neutrons. The average $30 \mathrm{keV}$ capture cross sections for these two sprocess nuclei were found to be $225 \pm 35 \mathrm{mb}$ for ${ }^{134} \mathrm{Ba}$ and $61 \pm 10 \mathrm{mb}$ for ${ }^{136} \mathrm{Ba}$.

* To be submitted for publication in Nuclear Physics.

** Relevant to request No. 72072.

+ Nuclear Physics A256, 193 (1976). 


\section{Ł. keV Neutron Resonance Capture in Barium-135 * (Musgrove, Allen and MackTin)}

The neutron capture cross section of ${ }^{135} \mathrm{Ba}$ has been measured with high resolution at the Oak Ridge Electron Linear Accelerator in the energy range 3 to $100 \mathrm{keV}$. From over ninety observed resonances in the 3 to 6 $\mathrm{keV}$ energy range, the average resonance parameters obtained were: $\left\langle\Gamma_{\rangle}\right\rangle=$ $150 \pm 20 \mathrm{meV} ;\left\langle\Gamma_{\gamma}\right\rangle=39.3 \pm 4 \mathrm{eV}$ and $10^{4} \mathrm{~S}_{1}=0.8 \pm 0.2$. The quoted radiation width and $p$-wave strength function also have a normalization error of \pm 20 percent. The method of separation of $s$ - and $p$-wave populations by statistical methods is described.

\section{m. keV Neutron Resonance Capture in ${ }^{138} \mathrm{Ba}$ ** (Musgrove, Allen, Boldeman and Macklin)}

The neutron capture cross section of ${ }^{138} \mathrm{Ba}$ has been measured with high resolution to $100 \mathrm{keV}$ and resonance parameters have been extracted. A number of new levels are observed. The $s$-wave radiative width is found to be six times the $p$-wave radiative width and is also considerably larger than for other nuclei in this mass region. Valence capture and enhanced decay to single-particle final states could account for the large s-wave radiative strength. A large positive correlation between $\Gamma_{0}{ }^{0}$ and $\Gamma_{\gamma}$ for ten $s$-wave levels was found $(\rho=0.67)$. The following average resonance parameters were deduced: $\angle D\rangle=7.5 \pm 1.5 \mathrm{keV}, \mathrm{S}_{0}=(0.9 \pm 0.4) \times 10^{4}$, $S_{1} \approx 0.5 \times 10^{-4} ;<\Gamma_{s}=310 \pm 25 \mathrm{meV}$ and $\left\langle\Gamma_{p_{p}}>_{p}=47 \pm 5 \mathrm{meV}\right.$. Evidence for a predominant direct capture mechanism for thermal capture is presented.

$$
\text { n. The }{ }^{165} \mathrm{Ho}(n, \gamma) \text { Standard Cross Section from } 3 \text { to } 450 \mathrm{keV}+
$$

The ${ }^{165} \mathrm{Ho}(n, \gamma)$ cross section was measured at the Oak Ridge Electron Linear Accelerator neutron time-of-flight facility. Nonhydrogenous scintillation detectors were used with pulse-height weighting to measure the prompt photon yield, normalized to the saturated 3.92-eV resonance in $\left({ }^{165} \mathrm{Ho}+n\right)$ and the shape of the ${ }^{6} \operatorname{Li}(n, \alpha)$ cross section. Resonance parameters for many of the observed peaks below $3 \mathrm{keV}$ were determined by a nonlinear least-squares fit. The data to $100 \mathrm{keV}$ were well fitted with energy-independent strength functions $10^{4} \mathrm{SO}=1.33 \pm 0.14,10^{4} \mathrm{~S}^{1}=1.36 \pm$ $0.24,10^{4} S_{2}=1.19 \pm 0.76$ and $\bar{\Gamma}_{2} / D_{0}=0.076 /(3.23 \pm 0.55 \mathrm{eV})$. The fluctuations of the cross section about the strength function fit are analyzed for 250-eV averages. The Wald-Wolfowitz "Runs" test is consistent with no additional nonrandom structure in the cross section.

* $\operatorname{INDC}($ AUL $)-23 / \mathrm{L}$.

* Nuclear Physics, to be published.

+ Nucl. Sci. Eng. 59, 231 (1976). 
o. Gold Neutron-Capture Cross Section from 3 to $550 \mathrm{keV}$ *, **

(R. L. Macklin, J. Halperin and R. R. Winterst)

A careful remeasurement of the ${ }^{197} \mathrm{Au}(n, \gamma)$ cross section using the pulse height weighting technique in small scintillators has been completed. The 4.9-eV resonance was used for calibration and the ${ }^{6} \mathrm{Li}(\mathrm{n}, \alpha)$ cross $\mathrm{sec}-$ tion for flux shape. Estimated errors range from 1.4\% near $30 \mathrm{keV}$ to $3.3 \%$ at $550 \mathrm{keV}$. Individual resonance parameters were deduced in the 2.6-4.9 $\mathrm{keV}$ range and the fluctuations over 10's of resonances were analyzed below $90 \mathrm{keV}$. The fluctuations are larger than expected, limiting the precision attainable with monoenergetic sources using this standard. The fluctuation intensity appears to indicate intermediate resonance structure in the compound nucleus with $\sim 10 \mathrm{keV}$ width and $\sim 40 \mathrm{keV}$ spacing.

\section{p. Stellar Neutron Capture in the Thallium Isutopes中 (R. L. Macklin and R. R. Winters)}

High resolution meulron capture cross section data for isotopically enriched samples of ${ }^{203} \mathrm{Tl}$ and ${ }^{205} \mathrm{Tl}$ were taken at the Oak Ridge Electron Linear Accelerator time-of-flight facility. The resonance parameter data were used to calculate neutron capture probabilities over a range of stellar interior temperatures. A semi-empirical estimate is also interpolated for the radioactive ${ }^{204} \mathrm{Tl}$ and ${ }^{205} \mathrm{~Pb}$ and the results used to recalculate the time scale of s-process nucleosynthesis.

\section{q. The Neutron Strength Functions and Radiation Widths of ${ }^{206} \mathrm{Tl}$ Resonances $\pi$ (Winters, Earle, $\$$ Harvey and Macklin)}

The parameters of ${ }^{206} \mathrm{Tl}$ resonances have been determined from neutron total cross section measurements and neutron capture measurements on the Oak Ridge Electron Linear Accelerator.

The neutron strength function $\Sigma g \Gamma_{n}^{0} / \Delta E$ for known $s$-wave resonances is $0.2 \times 10^{-4}$ below $50 \mathrm{keV}$ and $1.1 \times 10^{-4}$ above $50 \mathrm{keV}$. The strength function $\Sigma g \Gamma_{n}^{1} / \Delta E$ for known $p$-wave resonances is $0.5 \times 10^{-4}$ below $65 \mathrm{keV}$ and $1.5 \times 10^{-4} 4$ above. The contribution to these strength functions of unidentified resonances is ton small to modify the conclusion that both exhibit a change of slope at around $60 \mathrm{keV}$. This effect could be interpreted as evidence for intermediate structure (as has previously been $\operatorname{seen}^{1}$ ) for $s$-wave resonances in the $\mathrm{Pb}$ isotopes; however, it is seen for both $s$ - and $p$-wave resonances at about the same energy.

* Phys. Rev. C 11, 1270 (1975).

** Relevant to request Nos. 67082 and 72073.

+ Denison University.

₹ To be published in Journal of Astrophysics.

$\pi$ Abstract of paper to be presented at 1976 Int. Conf. Interactions of Neutrons with Nuclei, Lowell, Massachusetts, 6-9 July 1976.

\$ Chalk River Nuclear Laboratory, AECL, Chalk River, Ontario.

1 H. W. Newson, Statistical Properties of Nuclei, Ed. J.B. Garg, Plenum Press (1972) p. 309 and references cited therein. 
The radiative widths fall into two groups depending on the parity of the resonances. The $s$-wave resonances have $\Gamma_{\gamma}$ in the range 0.8 to $6 \mathrm{eV}$ while the $p$-wave resonances have $\Gamma_{\gamma}$ in the range 0.04 to $0.2 \mathrm{eV}$. It is suggested that this unusual separation of radiative widths into two families is due to the nature of the low lying states. Since all known states below $2 \mathrm{MeV}$ have high spin or negative parity there can be no high energy El primaries from $p$-wave resonances whereas many El transitions from each s-wave resonance are possible.

\section{r. Resonance Neutron Capture by ${ }^{209} \mathrm{Bi} *$ (R. Mackl in and J. Halperin)}

Neutron capture measurements were made for bismuth samples up to the inelastic scattering threshold at $90 \mathrm{i} \mathrm{keV} \mathrm{(lab).} \mathrm{Ail} \mathrm{resonance} \mathrm{peaks}$ $(l=0.1)$ between $2.6 \mathrm{keV}$ and $30 \mathrm{keV}$ were fitted to single level parameters by least squares adjustment. From 30 to $70 \mathrm{keV}$ the resonances reported from recent neutron transmission studies were fitted. Average radiative widths found were $(164 \pm 45) \mathrm{meV}$ for $\ell=0$ and $(33.7 \pm 3) \mathrm{meV}$ for $\ell=1$. The average spacing of p-wave levels was $1.14 \pm .25 \mathrm{keV}$ for the energy interval $2.6-30 \mathrm{keV}$. The astrophysical average capture $(10.7 \mathrm{mb}$ at $\mathrm{kT}=$ $30 \mathrm{keV}$ ) is little different from earlier estimates but the rate of change with stellar temperature is slower.

\section{Total Cross Sections}

a. Measurement of the Neutron Total Cross Section of Sodium ${ }^{\star *}$, + (D. C. Larson, J. A. Harvey and N. W. Hill)

Recent sensitivity analyses ${ }^{1}$ for the CRBR upper axial shield indicate that $40 \%$ of the integrated tissue dose sensitivity to the sodium total neutron cross section comes from the interference minimum of the 300-keV resonance. With the large quantities of liquid sodium coolant present in the CRBR, the cross section minimum for this resonance takes on new significance. Recent thick sample measurements on sodium minima by Brown et $a l^{2}$ show a significant discrepancy with the present ENDF/B-IV evaluation ${ }^{3}$ for the $300-\mathrm{keV}$ resonance. The evaluation in this region is

* To be submitted for publication in Phys. Rev. C.

** Abstract of paper to be presented at 1976 Int. Conf. Interactions of Neutrons with Nuclei, Lowe11, Massachusetts, 6-9 July 1976.

† Relevant to request Nos. 74010 and 74011 .

1 E. M. Oblow, "Survey of Shielding Sensitivity Analysis Development and Applications Program at ORNL," ORNL-TM-5176 (January 1976).

2 P. H. Brown, B. L. Quan, J. J. Weiss and R. C. Block, Trans. Am Nucl. Soc. 21,505 (1975).

${ }^{3}$ N. C. Paik and T. A. Pitterle, "Evaluation of Sodium-23 Neutron Data for the ENDF/B Version III File," Appendix A, WARD-3045T4B-2, Westinghouse Advanced Reactors Division (April 1972), and ENDF/B-IV, MAT 1156, NNCSC, Brookhaven National Laboratory (1974). 
based on the data of Cierjacks et al. ${ }^{l}$ which show a much sharper minimum than revealed by the measurement of Brown. In addition to the 300-keV resonance problem, no high resolution total cross section data were available from $\sim 40 \mathrm{keV}$ to $300 \mathrm{keV}$, leading to large uncertainty estimates for this energy region in the evaluation.

In order to provide consistent high resolution data for the ENDF/B$\checkmark$ evaluation, as well as to verify the high resolution data of Cierjacks, we have measured the transmission of neutrons through a $8.1-\mathrm{cm}(1 / \mathrm{n}=4.90$ b/atom) sample of pure sodium from $40 \mathrm{keV}$ to $20 \mathrm{MeV}$. The transmitted beam was detected by a NE-110 proton recoil detector located at the 200-m flight path of the Oak Ridge Electron Linear Accelerator (ORELA). A 5-ns electron beam burst width was used, with a repetition rate of $800 \mathrm{sec}^{-1}$. The data were corrected for dead-t.ime effects (maximum of $9 \%$ in the cross section at $1.1 \mathrm{MeV}$ ), and background (varying from $0.1-0.2 \%$ between $100 \mathrm{keV}$ and $2 \mathrm{MeV}$, rising to $1 \%$ at $8 \mathrm{MeV}$ ). The 50,000 channels of transmission data were suitably averaged to improve counting statistics while preserving the resonance structure and were then converted to cross section versus energy.

We observe eight resonances in the region of 190 to $310 \mathrm{keV}$, four of which have not been seen in previous transmission measurements. Resonance energies are $201.2,214,236.8$, *239.5, 243.1,* 298.1, 299.4* and $305.2 * \mathrm{keV}$, where the asterisk labels the new resonances. The present data near the $300-k e V$ minimum agree well with data of Brown et al. The largest deviation of the present data from the evaluation in the vicinity of the minimum is $-12 \%$.

In summary, the present measurement points out several areas for improvement in the sodium evaluation for ENDF/B-V, the most important being the broadening of the minimum at $300 \mathrm{keV}$.

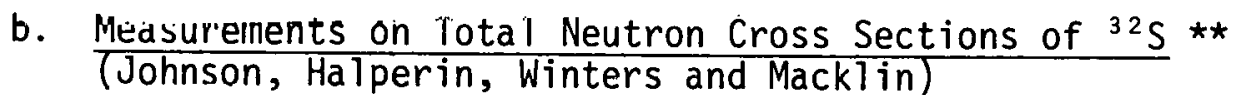

In the valency model for neutron capture enhanced dipole transitions occur from states with relatively large neutron widths to final states with large neutron spectroscopic factors. In order to study this phenomenon we need to know not only $\Gamma_{\gamma}$ for each resonance but also the neutron widths and JT-values. The observed total cross sections give the neutron width and J-values for well-resolved resonances. The parity can also be deduced if the non-resonant. phase shift is large enough to yive àn interference pattern.

1 C. Cierjacks, P. Forti, D. Kopsch, L. Kropp, J. Neve and H. Unseld, "High Resolution Total Neutron Cross Sections Between 0.5 to $30 \mathrm{MeV}$," Karlsruhe report KFK-1000 (June 1968).

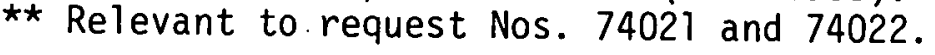


We have measured the neutron total cross section of ${ }^{32} \mathrm{~S}$ using the $200 \mathrm{~m}$ flight path with $5 \mathrm{nsec}$ pulses at ORELA to supplement our earlier neutron capture data obtained at the 40 meter station. From these data we have determined $\Gamma_{n}$ and $J^{\pi}$ for 15 of the $\sim 60$ resonances observed in capture. There is no obvious correlation of $\Gamma_{n}$ and $\Gamma_{\gamma^{*}}$. But the radiative widths predicted from the valency model from the observed $\Gamma_{n}$ and $J^{\pi}$ are large fractions of the observed $\Gamma_{\gamma}$. Furthermore, resonances predicted to have relatively large valency capture are observed to have relatively intense high energy gamma rays in the emitted spectra.

\section{c. Neutron Total Cross Sections and Resonance Parameters of ${ }^{6}{ }^{3} \mathrm{Cu}$ and $65 \mathrm{Cu}$ * (Pandey, Garg and Harvey)}

The neutron total cross sections of the isotopes of copper have been measured with high resolution using the ORELA neutron facility. From the area and shape analys is of the transmission and total cross section data, precise values of the resonance parameters, such as $E_{0}$, $\Gamma_{n}^{0}, \Gamma_{n}^{1}, J^{\pi}$, etc., have been determined. For example, for ${ }^{63} \mathrm{Cu}$ many $s$-wave resonances have been observed from 10 to $150 \mathrm{keV}$ giving values of $\langle D\rangle_{\mathrm{J}=1}=(2.7 \pm 0.3) \mathrm{keV},\langle\mathrm{D}\rangle_{\mathrm{J}=2}=(4.0 \pm 0.5) \mathrm{keV},\langle\mathrm{D}\rangle_{\mathrm{J}=1+2}=(1.63 \pm 0.13) \mathrm{keV}$, $\mathrm{S}_{\mathrm{O}_{\mathrm{J}=1}}=(2.8 \pm 0.6) \times 10^{-4} \mathrm{eV}^{-1 / 2}, \mathrm{~S}_{\mathrm{J}=2}=(1.9 \pm 0.5) \times 10^{-4} \mathrm{eV}^{-1 / 2}, \mathrm{~S}_{\mathrm{O}_{\mathrm{J}=1,2}}=$ $(2.4 \pm 0.4) \times 10^{-4} \mathrm{eV}^{-1 / 2}$. For ${ }^{65} \mathrm{Cu} s$-wave resonances were observed giving values of $\langle D\rangle_{J=1}=(3.6 \pm 0.4) \mathrm{keV},\langle D\rangle_{J=2}=(5.2 \pm 0.7) \mathrm{keV},\langle D\rangle_{J=l}+2=(2.12 \pm 0.19)$ $\mathrm{keV}, \mathrm{S}_{\mathrm{O}_{\mathrm{J}=1}}=(2.8 \pm 0.6) \times 10^{-4} \mathrm{eV}^{-1 / 2}, \mathrm{~S}_{\mathrm{O}_{\mathrm{J}=2}}=(1.8 \pm 0.5) \times 10^{-4} \mathrm{eV}^{-1 / 2}, \mathrm{~S}_{\mathrm{O}_{\mathrm{J}=1,2}}=$ $(2.3 \pm 0.4) \times 10^{-4} \mathrm{eV}^{-1 / 2}$.

d. Resonances in $207 \mathrm{~Pb}+n$ Including $\mathrm{d}+\mathrm{s}$ Wave Admixture ${ }^{\star *}$ (Horen, Harvey and $\mathrm{Hill}$ )

High resolution ( $\sim 0.07 \%$ ) neutron transmission data of ${ }^{207} \mathrm{~Pb}$ (enriched to $92.4 \%$ ) taken at the 200-meter flight path at ORELA are being analyzed to determine resonance parameters. Of particular interest is the observation of a large $d$ - and s-wave admixture in the 256.25-keV resonance $\left(\mathrm{J}^{\pi}=1^{-}, \Gamma=3.2 \mathrm{keV}\right)$. Analys is of this resonance assuming only s-wave contribution leads to an abnormally large radiation width, $\Gamma_{\gamma} \approx 1 \mathrm{keV}$. The resonance was therefore analyzed usjing a 2-channel, single-level, Rmatrix formalism which gave $\Gamma_{n}^{d}=0.71 \Gamma_{n}^{S}$. This result is consistent with photonuclear polarization and angular distribution studies. ${ }^{1}$ Similar analyses indicate that the $101.78-\mathrm{keV}\left(\mathrm{J}^{\pi}=1^{-}, \Gamma=75 \mathrm{eV}\right)$ resonance also contains a d-wave component. These d-wave components correspond to a few percent of the Wigner limit. A neutron scattering experiment at the

* Submitted for publication in Phys. Rev.

** Relevant to request No. 74315 .

1 R. Holt, private communication, January 1976. 
200-meter flight path is in progress. Preliminary data show that the shapes of a number of resonances are strongly angular dependent, and indicate such measurements will be valuable in assigning unambiguous $\ell$-values. Our results indicate numerous discrepancies with previously reported values.

\section{e. Resonant States of ${ }^{209} \mathrm{~Pb}$ from Neutron Total Cross Section Measurements* (Fowler, Johnson and Hill)}

By the use of a suitable combination of three natural samples of lead in a transmission experiment we have measured the total neutron cross section of effectively $99.6 \%{ }^{208} \mathrm{~Pb}$. The Oak Ridge Electron Linear Accelerator, ORELA, time-of-flight facility provided 4-5 ns pulses of neutrons for the 200 meter flight path. We observed 2100 resonances between .7 MeV and $1.5 \mathrm{MeV}$, about twice as many as were seen in a previous experiment ${ }^{1}$ carried out with ${ }^{7} \operatorname{Li}(p, n)$ neutrons. In this energy interval our resolution varied from $2.6 \mathrm{keV}$ at $0.7 \mathrm{MeV}$ to $27.4 \mathrm{keV}$ at $1.5 \mathrm{MeV}$. The improved energy resolution and statistics from this experiment together with differential cross sections from the previous experiment $^{1}$ will enable us to identify the $J$ values, parities and reduced widths of the resonant states of $20{ }^{\circ} \mathrm{Pb}$ more definitcly than was possible earlier. In the case of the other doubly closed shell nuclei such data give information of the fragmentation of shell model states. ${ }^{2}$

\section{Scattering and Reactions}

a. Angular Anisotropy in the ${ }^{6} \mathrm{Li}(n, \alpha)$ T Reaction** ${ }^{* *}$ (Harvey,

The yield of tritons and alphas in forward and backward directions $(\Omega \approx \pi)$ from the interaction of neutrons with ${ }^{6} \mathrm{Li}$ has been measured from a thin sample of ${ }^{6} \mathrm{LiF}\left(101 \mu \mathrm{gm} / \mathrm{cm}^{2}\right)$ from 0.5 to $25000 \mathrm{eV}$. The measurements were made with a diffused junction $\mathrm{Si}$ detector located 9.03 meters from the water-moderated Ta-target at ORELA. The alpha and triton groups at 2.06 and $2.73 \mathrm{MeV}$ are well resolved enabling one to obtain an accurate ratio of their intensity as a function of neutron energy. The intensity of the alpha peak was greater than the intensity of the triton peak in lhe forward direction but less in the backward direction. This difference is measureable down to $10 \mathrm{eV}$. An anisotropy has already been reported for.

* Relevant to request No. 74315 .

1 J. L. Fowler, Phys. Rev. 147, 870 (1966).

2 J. L. Fowler, C. H. Johnson and R. M. Feeze1, Phys. Rev. C 8, 545 (1973) and C. H. Johnson, Phys. Rev. C 7,561 (1973).

** Relevant to request Nos. 69009-11. 
$25 \mathrm{keV}$ neutrons by Schroder et al. ${ }^{1}$ who made measurements using an ironfiltered neutron beam. In the $10 \mathrm{eV}$ to $10 \mathrm{keV}$ energy region, our results give an energy dependence for the alpha to triton ratio at $180^{\circ}$ (or triton to alpha ratio at $0^{\circ}$ ) of the form $1+C E^{0.54}$ where $c$ is 20.005 and $E$ is the neutron energy in eV. At $100 \mathrm{eV}$ this ratio equals 1.06 . It is necessary to consider this angular anisotropy when the ${ }^{6} \mathrm{~L} i(n, \alpha)$ cross section is used as a standard. This angular anisotropy probably arises from the interference between the large p-wave resonance at $245 \mathrm{keV}$ and many $s$-wave resonances which account for the large $1 / v(n, \alpha)$ thermal cross section.

b. High Resolution Neutron Scattering Experiments at ORELA* (W. E. Kinney, J. W. McConne1T and T. A: Love)

Neutron elastic and inelastic scattering data taken with $0.2-0.3$ $\mathrm{nsec} / \mathrm{m}$ resolutions have been reduced to cross sections from 500 to 3000 - keV in 1-keV intervals. The results include: 1) $\mathrm{Na}, \mathrm{Si}$, and Fe differential $(n, n)$ cross sections at 8 angles from $24^{\circ}$ to $155^{\circ}$ relative to $C$; 2) $\mathrm{Na}$ and Fe differential $\left(n, n^{\prime} \gamma\right)$ cross sections at $30^{\circ}, 90^{\circ}$, and $125^{\circ}$ relative to the ${ }^{7} \mathrm{Li} 478-\mathrm{keV} \gamma$-production cross section; 3 ) $\mathrm{Na}, \mathrm{Al}, \mathrm{Si}$, $V$, and $\mathrm{Fe}\left(n, n^{\prime} \gamma\right)$ measured with $\sim 30 \% 4 \pi$ geometry relative to the ${ }^{7} \mathrm{Li}$ 478-keV $\gamma$-production cross section.

C. Cross Sections for the $A l(n, x n)$ and $A](n, x y)$ Reaction between 1
and $20 \mathrm{MeV}^{\star *}, t$. (G. L. Morgan and F. G. Perey)

Differential cross sections for the production of secondary neutrons and photons from aluminum have been measured at $127^{\circ}$ ( $1 \mathrm{ab}$ ) for incident neturon energies in the range 1 to $20 \mathrm{MeV}$. An electron linac was used as a neutron source with a white spectrum. Incident neutron energies were determined using time-of-flight techniques for a source-tosample distance of $48 \mathrm{~m}$. Secondary spectra were determined by unfolding the pulse-height distributions observed in a NE-213 scintillation counter. The results are compared to the current evaluated data file (ENDF/B-IV, MAT 1193).

I I. G. Schroder, E. D. McGarry, G. deLeeuw-Gierts and S. deleeuw, Conference on Neutron Cross Sections and Technology, NBS Special Publication 425 (1975) p. 240.

* Relevant to request Nos. 74012-13, 62007, 69045, 69084-87, 66016-17.

** Abstract of ORNL-TM-5241; paper submitted for publication in Nucl. Sci. Eng.

+ Relevant to request Nos. 74162-63, 74215, 17268-69. 
d. $\frac{(n, \alpha),(n, p),(n, \gamma) \text { and Total Neutron Cross Section Measurements }}{\text { upon 59Ni (Harvey, Halperin, Hill, Raman and Macklin) }}$

In addition to $(n, \gamma)$ and total neutron cross section measurements upon ${ }^{59} \mathrm{Ni}$ we have made $(n, \alpha)$ and $(n, p)$ measurements at ORELA from $20.01 \mathrm{eV}$ to $\sim 20 \mathrm{keV}$. The thermal and resonance $(n, \alpha)$ cross sections of this isotope which are produced from ${ }^{58} \mathrm{Ni}(n, \gamma)$ are important due to helium embrittlement and swelling of the structural material of power reactors.

The $(n, \alpha)$ and $(n, p)$ measurements were made simultaneously with a diffused junction Si detector located 9.03-meter from the water-moderated neutron target at ORELA resulting in a neutron energy resolution of $0.5 \%$

(FWHM). A sample of ${ }^{59} \mathrm{Ni}\left(95 \%, 91 \mu \mathrm{gms} / \mathrm{cm}^{2}\right)$ was electroplated upon a $1-\mathrm{mil}$ Pt foil and a deposit of ${ }^{6} \mathrm{Li}\left(95 \%, 104 \mu \mathrm{gm} / \mathrm{cm}^{2}\right)$ was evaporated on top of this ${ }^{5} \mathrm{Ni}$ depos1t. The triton and alpha groups $(2728$ and $2056 \mathrm{keV}$ for thermal neutrons) from the ${ }^{6} \mathrm{~L} i(n, \alpha)$ reaction were easily resolved from the $4759-\mathrm{keV}$ alpha group from the ${ }^{59} \mathrm{Ni}(n, \alpha)$ reaction. The ${ }^{59} \mathrm{Ni}(n, p)$ measurements were made with a deposit of only ${ }^{59} \mathrm{Ni}$ since the $1827-\mathrm{keV}$ protons were difficult to separate from the alphas from ${ }^{6} \mathrm{Li}$.

The $(n, \gamma)$ cross section measurement was made with a 3.136-gram $\mathrm{Ni}$ sample $2.54-\mathrm{cm}$ dia. enriched to $2.96 \%$ in ${ }^{59} \mathrm{Ni}$. The measurements were made from 100 to $12000 \mathrm{eV}$ with an energy resolution of $0.15 \%$ using the total energy detector located at a 40-meter flight path. Since the sample contained a small amount of ${ }^{60} \mathrm{Co}$, the bias level on this gamma ray detector was set $22 \mathrm{MeV}$ involving an uncertainty of $10 \pm 5 \%$. Another measurement is planned with a "clean" sample to reduce the uncertainty due to this extrapolation.

Neutron total cross section measurements were also made with this $3.136-\mathrm{gm}$ sample with an inverse thickness of $5295 \mathrm{barns} / \mathrm{atom}$ of ${ }^{59} \mathrm{Ni}$ using an 80-meter flight path. The total cross section of the 203.4 resonance obtained with this sample was 1.32 times that obtained for earlier measurements using the 29 -mg, 95\%-enriched sample reported in last year's report. ${ }^{1}$ This $29-\mathrm{mg}$ sample has been emptied, reweighed on a microbalance and examined for oxygen by measuring the ${ }^{16} \mathrm{~N}$ activity produced by the ${ }^{16} 0(n, p)$ reaction with $14-\mathrm{MeV}$ neutrons. These measurements confirmed the neutron analysis that the original sample contained only $7.0 \mathrm{mg}$ of $\mathrm{Ni}$ rather than $9.2 \mathrm{mg}$. This increases the thermal capture and absorption cross sections reported last year to $70 \pm 5$ and $87 \pm 6$ barns respectively.

The measurements on ${ }^{59} \mathrm{Ni}$ in the thermal energy range yield a value of $12 \pm 1$ barns for the $(n, \alpha)$ cross section and $2.0 \pm 0.5$ for the $(n, p)$ cross section. This $(n, \alpha)$ result is to be compared with earlier

'S. Raman, E. T. Jurney, J. A. Harvey and N. W. Hill, NCSAC Report (1975). 
values of $13.7 \pm 1.2,18.0 \pm 1.6$, and $22.3 \pm 1.6$ barns reported by Eiland and Kirouac, ${ }^{1}$ by Werner and Santry, ${ }^{2}$ and by McDonald and Sjöstrand ${ }^{3}$ respectively. The $(n, p)$ result is lower than the value of $4 \pm 1$ barns reported by McDonald and Sjöstrand. The results of our four experiments have been combined to produce the following parameters for the 203.4-eV resonance $E_{0}=203.4 \pm 0.2 \mathrm{eV}, \Gamma=13.3 \pm 0.2 \mathrm{eV}, \mathrm{g}=3 / 8, \mathrm{~J}=1, \Gamma_{\mathrm{n}}=$ $8.50 \pm 0.15 \mathrm{eV}, \Gamma_{\gamma}=4.0 \pm 0.6 \mathrm{eV}, \Gamma_{\alpha}=0.50 \pm 0.03 \mathrm{eV}$ and $\Gamma_{p}=0.063 \pm$ $0.006 \mathrm{eV}$. Assuming that the thermal cross sections arise mainly from the large 203.4-eV resonance, the $(n, p)$ thermal cross section would be expected to be $1 / 8$ the $(n, \alpha)$ cross section or 1.5 barns.

Fifteen higher energy resonances have been observed in the four types of experiments. The alpha and proton widths of the resonances vary widely due to selection rules and because they are essentially single channel processes to the ground state. For example, for the $2^{-}$ resonance at $3203 \mathrm{eV}, \Gamma_{\alpha}$ is $\varsigma_{0.01} \Gamma_{\mathrm{p}}$ but for the $1^{-}$resonance at 203.4 $\mathrm{eV}$ and the 9103-eV resonance, $\Gamma_{\alpha}$ is $\gtrsim 7 \Gamma_{\mathrm{p}}$.

5. Capture $\gamma$-Rays

a. Determination of Parity of ${ }^{98}$ Mo Resonances With Low Energy $\gamma$ Rays

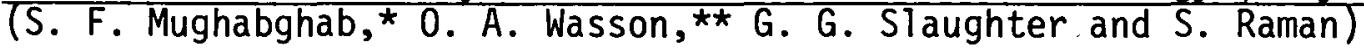

Low energy $\gamma$ rays were utilized to determine the spin and parity of neutron resonances. The spins of resonances at $429,467,613,1525$, $2178,2462,2947,3293,5432,5617 \mathrm{eV}$ were found to be $1 / 2$ while those at $12,402,818,1123,1922 ; 2565,2615,3264,3797,4013,4574,4861$, $5288 \mathrm{eV}$ are $\mathrm{J}=3 / 2$, and as a result are formed by $\mathrm{p}$-wave neutrons.

b. Neutron Interactions with ${ }^{100}$ Mo (Weigmann, Raman, Slaughter, Harvey, Mackl in and Halperin)

Neutron capture in the isotopes ${ }^{92}$ Mo and ${ }^{98}$ Mo is known to be dominated by the "valence capture" mechanism. Among these observations related to valence capture are strong correlations between reduced neutron widths of $\mathrm{p}$-wave resonances and partial radiation widths to final states with large $(d, p)$ spectroscopic factors such as the ground state. $10^{0}$ Mo was considered another candidate for valence capture, particularly because a strong concentration of p-wave strength occurs between 1 and $2.5 \mathrm{keV}$ neutron energy. Therefore, neutron capture $\gamma$-ray spectra were measured in separated resonances of $10^{\circ} \mathrm{Mo}$. As neutron widths given in

1 H. M. Eiland and G. J. Kirouac, Nucl. Sci. Eng. 53, 1 (1974).

R. D. Werner and D. C. Santry, Nucl. Sci. Eng. $5 \overline{6}, 98$ (1975).

3 J. McDonald and N. G. Sjöstrand, submitted to Atomkernenergie.

* Brookhaven National Laboratory, Upton, New York.

** National Bureau of Standards, Washington, D. C. 
the literature for the resonances in question are strongly discrepant, a transmission measurement was also performed to redetermine neutron widths. Simultaneously, total capture data, measured earlier with the ORELA neutron capture cross section measurement facility, have been analyzed.

The capture $\gamma$-ray spectra were measured with a $40 \mathrm{~cm}^{3} \mathrm{Ge}(\mathrm{Li})$ detector, using a $10.2 \mathrm{~m}$ flight path for neutron time-of-flight spectroscopy. Capture $\gamma$-ray spectra have been obtained for individual resonances up to about $5 \mathrm{keV}$ neutron energy. The transmission measurement has been performed at an $80 \mathrm{~m}$ flight path and resonance analysis is being done up to about $25 \mathrm{keV}$.

Althnugh analysis of the data is still in progress, two lli in results may already be given: (1) The neutron widths of the strnng $n-$ wave resonances between 1 and $2.5 \mathrm{kcV}$ neutron energy are considerably (up to a factor of three) smaller than those given in BNL-325. This reduces the probability that valence capture dominates and may in part explain the second observation; (2) Valence capture does not play a dominant role in these resonances: For instance, a transition to the ground state of ${ }_{101} \mathrm{Mo}$ (spectroscopic factor 0.42 ) is not observed in 3 of the 4 strongest p-wave resonances.

c. Neutron Capture Gamma-Ray Studies (S. Raman, G. G. Slaughter, R. F. Carlton, ${ }^{\star}$ J. C. Wells, Jr. ${ }^{\star}$ and D. A. McCluret)

The tin isotopes are well suited to a study of nuclear structure within the framework of the nuclear shell model because the magic number of protons $(Z=50)$ minimizes the need for considering $n-p$ pairing interactions in theoretical calculations and because the large number of stable isotopes makes it possible to study systematic trends in both experimental and she 11 model features. The existing experimental data on the odd-A tin isotopes are llul as extensive as one might expect on the basis of their theoretical importance. Thermal neutron capture studies have not been widely used due to the extremely small capture cross sections for the heavier even-A tin isotopes. Most experimental studies (especially nucleon transfer studies) are beset with the problem of interference from isotopic impurities. This usually necessitates an extensive study of all tin isotopes before conclusive results may be obtained. Resonance neutron capture offers a powerful technique for studying tin isotopes because interference from unwanted isotopes can be greatly suppressed through the combination of enriched targets and selection of resonances known to be in the nucleus under study. We have, therefore, undertaken a systematic investigation of the level structure of six odd tin isotopes between $A=115$ and $A=125$.

* Middle Tennessee State University, Murfreesboro.

** Tennessee Technological University, Cookeville.

+ Georgia Institute of Technology, Atlanta. 
Measurements have been completed on all except ${ }^{115} \mathrm{Sn}$ and ${ }^{117} \mathrm{Sn}$ which will be carried out in the near future. In the case of ${ }^{121} \mathrm{Sn}$, treated here as a typical case, capture $\gamma$-rays ( 18 primary and 32 secondary) from 16 neutron resonances up to $7 \mathrm{keV}$, obtained with a $\mathrm{Ge}(\mathrm{Li})$ detector, have been utilized to determine 20 excited levels in ${ }^{12}{ }^{1} \mathrm{Sn}$. Several new levels have been found. Spin and parity assignments have been made to many of the levels. When the present series of studies has been completed, the resulting level schemes for the tin isotopes are expected to significantly increase our understanding of their energy systematics.

We have also begun $(n, \gamma)$ measurements in the lead region aimed at obtaining information on absolute $\gamma$-ray transition widths and on reaction mechanisms. Measurements have been completed on enriched ${ }^{206} \mathrm{~Pb}$ and ${ }^{207} \mathrm{~Pb}$ targets. Unlike the case of the tin isotopes, the $\gamma$-ray spectra in lead isotopes are strikingly simple composed of less than 5 primary $\gamma$-ray transitions. The idea then would be to combine the relative $\gamma$-ray intensities obtained with a $\mathrm{Ge}(\mathrm{Li})$ detector (or even a $\mathrm{NaI}$ detector) with the total $\gamma$-ray widths obtained with a total energy detector in order to deduce the partial widths. In this manner, we have been able to determine the $E 2$ radiation widths for the ground state transitions in ${ }^{208} \mathrm{~Pb}$ from $2^{+}$neutron resonances at $3.1,10.2$ and $16.2 \mathrm{keV}$. The observed strengths represent only $\approx 0.3 \%$ of the energy-weighted sum-rule strength. By extending the $(n, \gamma)$ measurements to higher neutron energies, we hope to locate other pieces of the E2 strength as well as the El and MI strengths. Initial results obtained with a large $\mathrm{NaI}$ detector up to a neutron energy of $200 \mathrm{keV}$ are very encouraging.

d. Neutron Transmission and Capture Gamma-Ray Measurements of
$\frac{120 \mathrm{Sn}+n{ }^{\star}}{\text { Slaughter })}$ (R. F. Carlton, ${ }^{\star \star}$ S. Raman, J. A. Harvey and G. G.

Neutron transmission and neutron capture $\gamma$-ray measurements have been performed upon $98.45 \%$ enriched samples of ${ }^{12}{ }^{0} \mathrm{Sn}$. The transmission mea'surements were made at $18-\mathrm{m}$ and $80-\mathrm{m}$ neutron flight paths. Neutrons were detected by a ${ }^{6} \mathrm{Li}$ glass scintillator. Parameters have been obtained for 251 resonances up to $98 \mathrm{keV}$. From the shapes of the resonances, $\ell-$ value assignments have been made for $70 \%$ of the resonances. Level spacings and strength functions for $s$ - and $p$-wave neutrons have been obtained. Neutron capture $\gamma$ rays from 16 resonances, obtained with a $G e(L i)$ detector, have been utilized to determine the levels in ${ }^{121} \mathrm{Sn}$. New spin and parity assignments have been made for many of the levels. Four new levels have been found. The neutron separation energy was determined to be $6170.3 \pm 2: 0$ keV. The ${ }^{121} \mathrm{Sn}$ level scheme has been compared with those for ${ }^{11}{ }^{7} \mathrm{Sn}$ and ${ }^{119} \mathrm{Sn}$ to investigate systematic behavior.

* Submitted for publication in Phys. Rev.

** Middle Tennessee State University, Murfreesboro. 


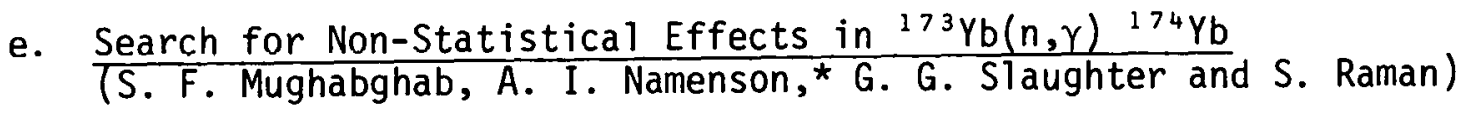

Capture $\gamma$ ray spectra due to neutron capture in an enriched ${ }^{173} \mathrm{Yb}$ sample were measured with a Ge(Li) detector at ORELA for the purpose of determining the correlation coefficient between partial radiative widths and neutron reduced widths. Spins of resonances were also determined.

6. Actinides

a. $\overline{\underline{v}}_{p}$ of ${ }^{252} \mathrm{Cf} * *$ (R. R. Spencer)

The most important parameter required in the design of nuclear power systems is $\bar{v}$ (the average number of neutrons emitted in fission) for the fisslie 1 sotonpes and its dependence on the encrgy of the neutrons inducing fission. Measurements of $\bar{v}_{p}(E)$ are most conveniently carried out as a ratio to $\bar{v}_{p}$ for spontaneous fission of $252 \mathrm{Cf}$. Therefore it is necessary to know the value of this standard to a high accuracy (of the order of $1 / 4 \%$ ). At ORNL measurements of $\bar{v}_{p}$ for ${ }^{252} \mathrm{Cf}$ are being pursued using a Gd-poisoned, liquid scintillator tank to detect the neutrons. Preliminary experiments have been carried out using the ORELA neutron source and an NE-213 proton recoil detector to "tag" neutrons scattered into the tank over a range of angles and neutron energies while simultaneously detecting the neutrons from a presently available (undocumented) ${ }^{252} \mathrm{Cf}$ source in a fission chamber. A three-dimensional data acquisition program, necessary for the proton recoil data, was developed by the computer section and successfully used in these experiments. The proton recoil data are being compared to Monte Carlo and Discrete Ordinate Transport (DOT) calculations of the tank absorption efficiency carried out by the neutron transport group at ORNL and to Monte Carlo calculations provided by a similar group at BAPL (Westinghouse). The calculations will be used to extend the measured detection efficiency over the full angular range of the tank and over the energy range necessary to describe the ${ }^{252} \mathrm{Cf}$ neutron spectrum. Support measurements of the tank time-of-absorption of neutrons have been performed in addition to a number of tests of the statistical consistency of the fast counting system. A new, low mass, fission chamber containing documented ${ }^{252} \mathrm{Cf}$ is under construction. A solid state proton recoil detector is being designed for use in the tank efficiency calibration at low neutron energies.

* U.S. Naval Research Laboratory, Washington, D. C.

** Relevant to request Nos. 69359, 72103 and 74130 . 
b. Neutron Energy Dependence of the Number of Prompt Neutrons Emitted in Fission of ${ }^{235} \mathrm{U}$ and ${ }^{239} \mathrm{Pu}$ * (Gwin, Ingle, Spencer, Todd and Weston)

Measurements of the neutron energy dependence of $\bar{v}$ the average number of prompt neutrons emitted per fission have been made on ${ }^{235} \mathrm{U}$ and $2{ }^{2} \mathrm{Pu}$ over the neutron energy range from about $0.005 \mathrm{eV}$ to a few tenths of an eV. In addition, a set of measurements was made for ${ }^{239} \mathrm{Pu}$ which extended to about $6 \mathrm{MeV}$. All of these measurements utilize ${ }^{252} \mathrm{Cf}$ as a standard.

Figure 1 shows the measured ratio of $\bar{v}$ for ${ }^{239} \mathrm{Pu}$ to that for ${ }^{252} \mathrm{Cf}$ over the neutron energy region from 0.003 to $0.3 \mathrm{eV}$. The increase in $\bar{v}$ for ${ }^{239} \mathrm{Pu}$, shown in $\mathrm{Fig}$. $\mathrm{I}$, as the energy decreases has been observed by Weinstein et al. ${ }^{1}$ Also shown in Fig. 1 is the measured ratio of $\bar{v}$ for ${ }^{235} \mathrm{U}$ to that for ${ }^{252} \mathrm{Cf}$. At the present level of the analysis, there is no demonstrated neutron energy dependence of $\bar{v}$ for ${ }^{235} \mathrm{U}$ over the energy region shown on Fig. 1. The background on the measurements shown in Fig. I was less than .05 cts compared to 3.14 cts for ${ }^{252} \mathrm{Cf}$.

The absolute value of the $\bar{v}$ ratios is of importance and the data shown in Fig. 1 for the ratio of $\bar{v}$ for ${ }^{239} \mathrm{Pu}$ to that for ${ }^{252} \mathrm{Cf}$ near $0.0253 \mathrm{eV}$ is about $0.6 \%$ higher than the value given by Lemme $1 .{ }^{2}$ The present experimental data have been corrected for the electronic dead time and preliminary calculations show that the correction for differences in the neutron fission spectrum is less than $0.1 \%$. The measured ratio of $\bar{v}$ for ${ }^{235} \mathrm{U}$ to that for ${ }^{239} \mathrm{Pu}$ for the neutron energy region about $0.02 \mathrm{eV}$ is within $0.1 \%$ of the value of 0.840 given by Lemmel. ${ }^{2}$

Two ${ }^{252} \mathrm{Cf}$ fission sources were used in the course of the present experiments and the effective $\bar{v}$ for these two sources differ by $0.25 \%$. It is not known at this time whether the disagreement results from contamination of either or both samples or whether the discrepancy results from fragment detection differences for the two chambers. The data reported here utilized the ${ }^{252} \mathrm{Cf}$ source which had the same physical geometry and electronics as the ${ }^{235} \mathrm{U}$ and ${ }^{239} \mathrm{Pu}$ samples, and the composition of this ${ }^{252} \mathrm{Cf}$ source is known. Use of the other 252 source, in a separately contained chamber, results in a lower value by $0.25 \%$ for the present measurements relative to ${ }^{252} \mathrm{Cf}$.

* Relevant to request Nos. 69250,69253-55, 66050,66066, 66069 and 66072 .

1 S. Weinstein, R. Reed and R. C. Block, "Neutron Multiplicity Measurements for ${ }^{23} \mathrm{U},{ }^{235} \mathrm{U}$ and ${ }^{2}{ }^{9} \mathrm{Pu}$ Resonance Fission," IAEA-SM-122/113.

$2 \mathrm{H}$. D. Lemmel, "The Third IAEA Evaluation of the $2200 \mathrm{~m} / \mathrm{sec}$ and $20^{\circ} \mathrm{C}$ Maxwellian Neutron Data for ${ }^{23}{ }^{3} \mathrm{U},{ }^{235} \mathrm{U},{ }^{239} \mathrm{Pu}$ and $241 \mathrm{Pu}, "$ Proc. Conf. Neutron Cross Sections and Technology, March 3-7, 1975, Washington, D.C. 


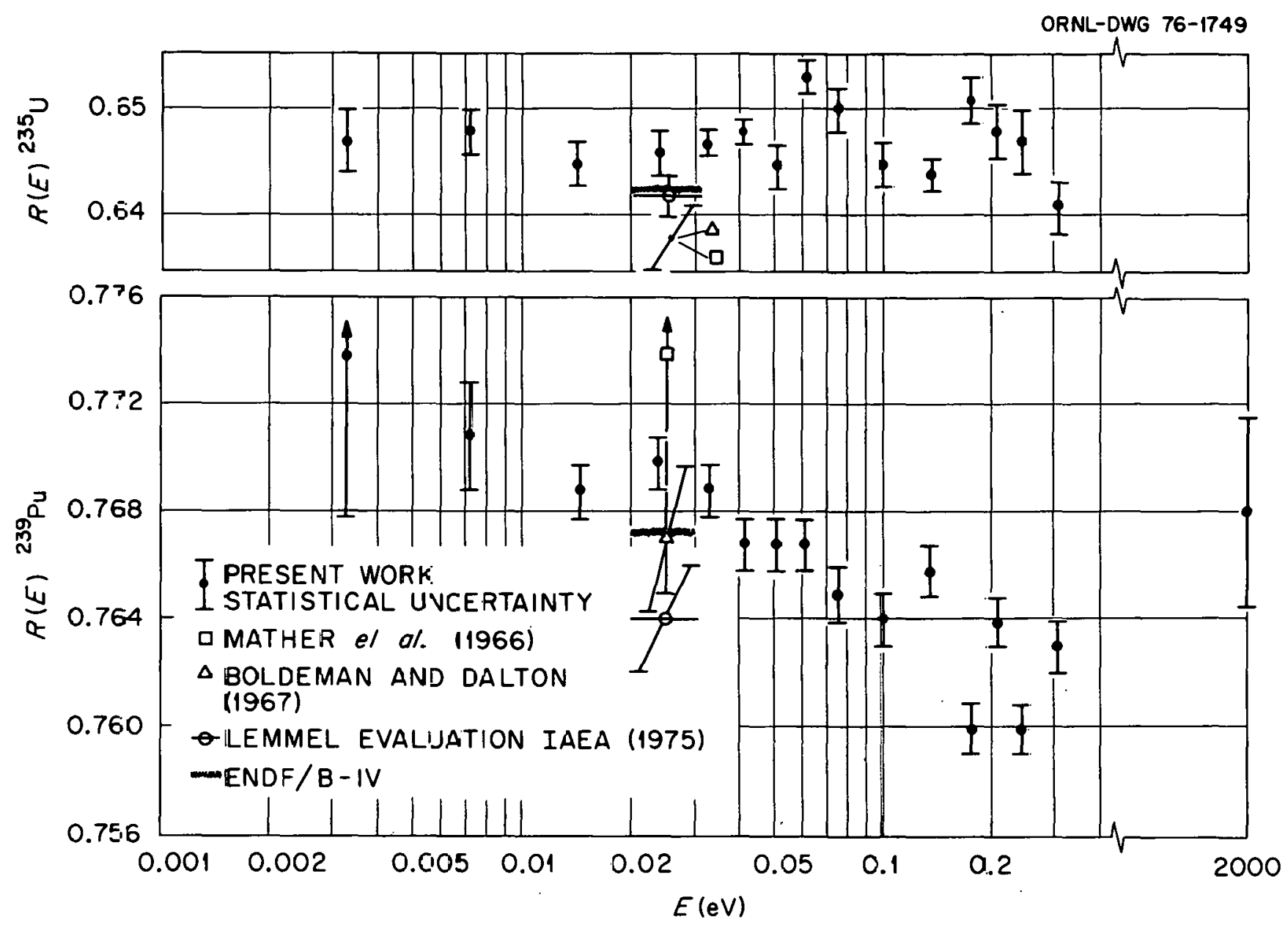

Fig. A-1. Energy [lependence $R(E)$ the Ratio of $\bar{\nu}_{p}$ for ${ }^{239} \mathrm{Pu}$ and ${ }^{235} \mathrm{U}$

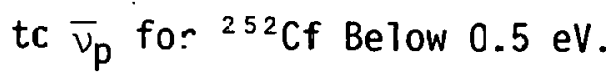


Figure A-2 shows a plot of $\bar{v}$ for $2{ }^{39} \mathrm{Pu}$ over the neutron energy range from 0 to $8 \mathrm{MeV}$. Also shown are the results obtained simultaneously for the ${ }^{252} \mathrm{Cf}$ Monitor. These experiments were performed at a $20 \mathrm{~m} \mathrm{flight} \mathrm{path} \mathrm{to} \mathrm{aid} \mathrm{in} \mathrm{establishing} \mathrm{operating} \mathrm{conditions} \mathrm{for} \mathrm{the}$ main experiments which are scheduled to be performed at an $85 \mathrm{~m}$ flight path. It is emphasized that the present results are preliminary.

c. Preliminary Fission Product Energy Release Measurements for Thermal Neutron Fission of $2.35 \mathrm{U}$ * (Dickens, Love, McConne 11, Emery and Peelle)

An experimental system to measure time-dependent spectra of beta and gamma rays from fission-product production by thermal neutron fission of ${ }^{235} \mathrm{U}$ is described, and for each component (beta and gamma) the system has been tested with a pilot data-accumulation run. Data reduction techniques are described and test results given. Gamma-ray spectra are compared with calculations using ENDF/B-IV data files. Both beta- and gamma-ray spectra were integrated to give total yields and total energy-release results for times after fission between 3 and 14400 sec. These preliminary integral data are compared with previous measurements and with integral calculations using ENDF/B-IV data files.

Figure A-3 shows $t f_{\gamma}(t)$ vs time after a fission, where $f_{\gamma}(t)$ is the gamma-ray energy emission rate $(\mathrm{MeV} / \mathrm{sec})$ following fission. Figure A-4 shows a sample photon spectrum compared to calculations based on known fission product yields and decay spectra.

d. Measurement of. the Neutron Capture and Fission Cross Sections of $239 \mathrm{Pu}$ and ${ }^{235} \mathrm{U}, 0.02 \mathrm{eV}$ to $200 \mathrm{keV}$, the Neutron Capture Cross Sections of ${ }^{19 \mathrm{Au}, 10}$ to $50 \mathrm{keV}$, and Neutron Fission Cross Sections of ${ }^{23} \mathrm{U}, 5$ to $200 \mathrm{keV}$ *t (Gwin, Silver, Ingle and Weaver)

The neutron absorption and fission cross sections for ${ }^{239} \mathrm{Pu}$ and $235 \mathrm{U}$ have been measured over the neutron energy range from $0.02 \mathrm{eV}$ to $200 \mathrm{keV}$. In addition, the neutron capture cross section for ${ }^{19}{ }^{7} \mathrm{Au}$ was measured from 10 to $50 \mathrm{keV}$ and the fission cross section of ${ }^{23} \mathrm{U}$ was measured from 0.1 to $100 \mathrm{keV}$. Normalization of the ${ }^{239} \mathrm{Pu}$ and ${ }^{235} \mathrm{U}$ data was made over the energy region from 0.02 to $0.4 \mathrm{eV}$ to the ENDF/B-III neutron cross sections for these isotopes, Mat 1159 and 1157 , respectively. The capture cross section for ${ }^{197} \mathrm{Au}$ was normalized using the saturated resonance method for the 4.9-eV resonance. For ${ }^{23} \mathrm{U}$ fission,

* Abstract of ORNL/TM-5273.

** Nuci. Sci. Eng. 59, 79 (1976).

+ Relevant to request Nos. 62037, 62039-40, 66043, 67089, 69226, 69241, $69245,69307,69439,69449,69452,69467,72085$ and 74207. 


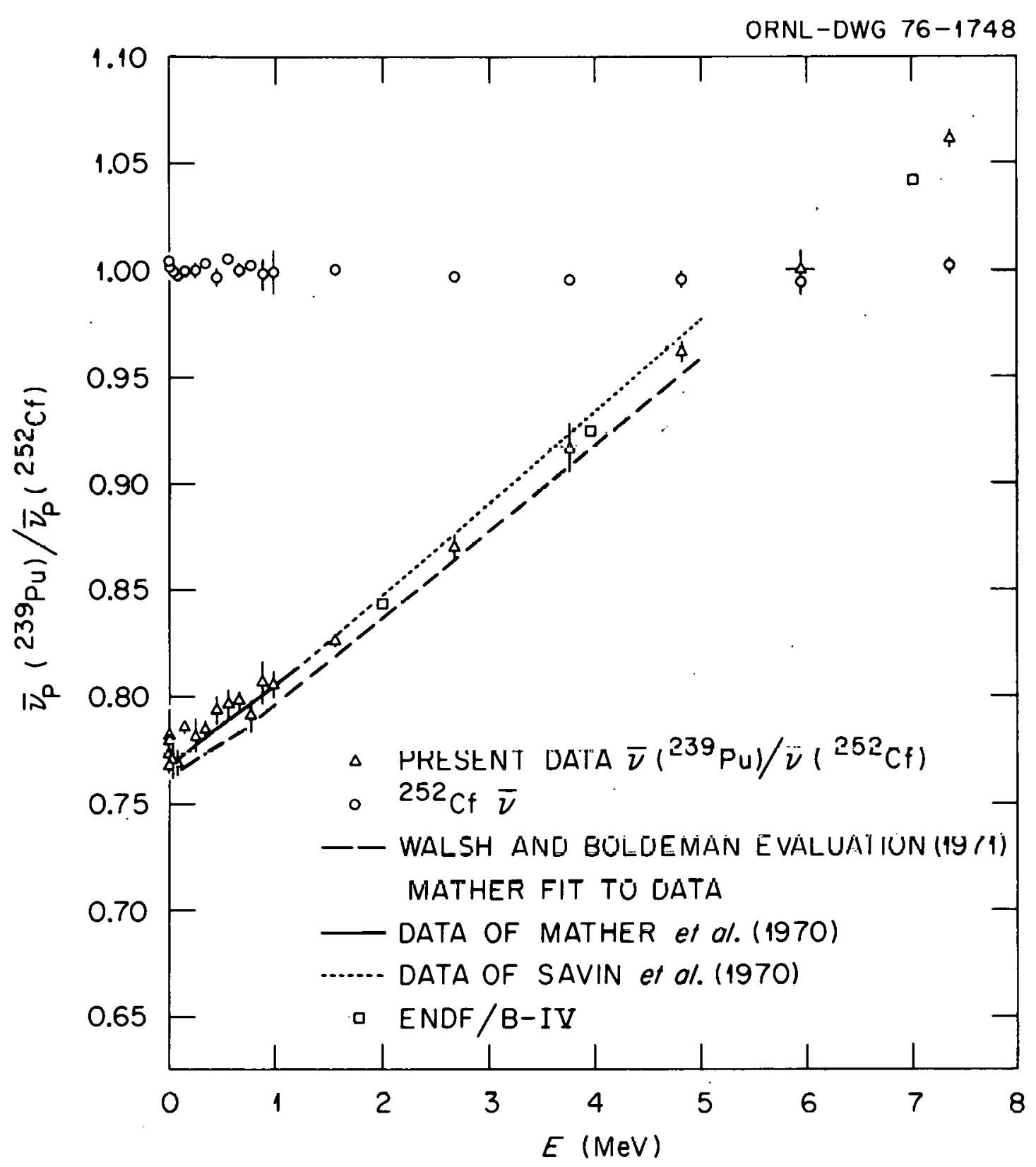

Fig. A-2. $\bar{\nu}_{p}{ }^{239 p u}$ Relative to $\bar{\nu}_{p}$ for ${ }^{252} \mathrm{Cf}$ vs Energy, 0 to $8 \mathrm{MeV}$. 
ORNL-OWG 76-2031

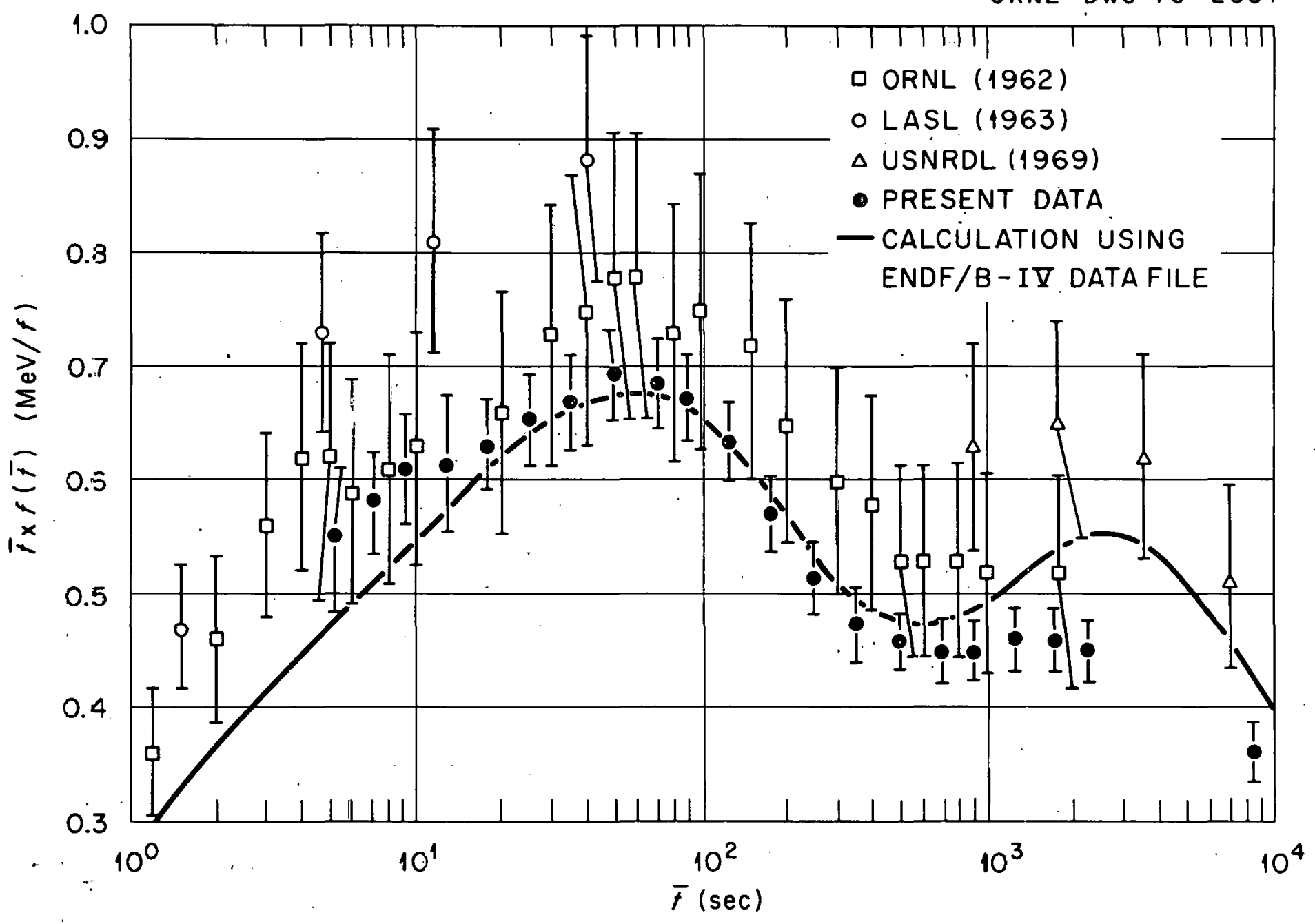

Fig. A-3. Photon Energy Emission Rate for Thermal-Neutron Fission of $235 \mathrm{U}$. The open squares are the data of Peelle, et al., open circles represent data of Fisher and Engle, and the open triangles are data of Bunney and Sam. The calculation was carried out by R. Schenter (Hanford) using the RIBD code and the ENDF/B-IV data file. 


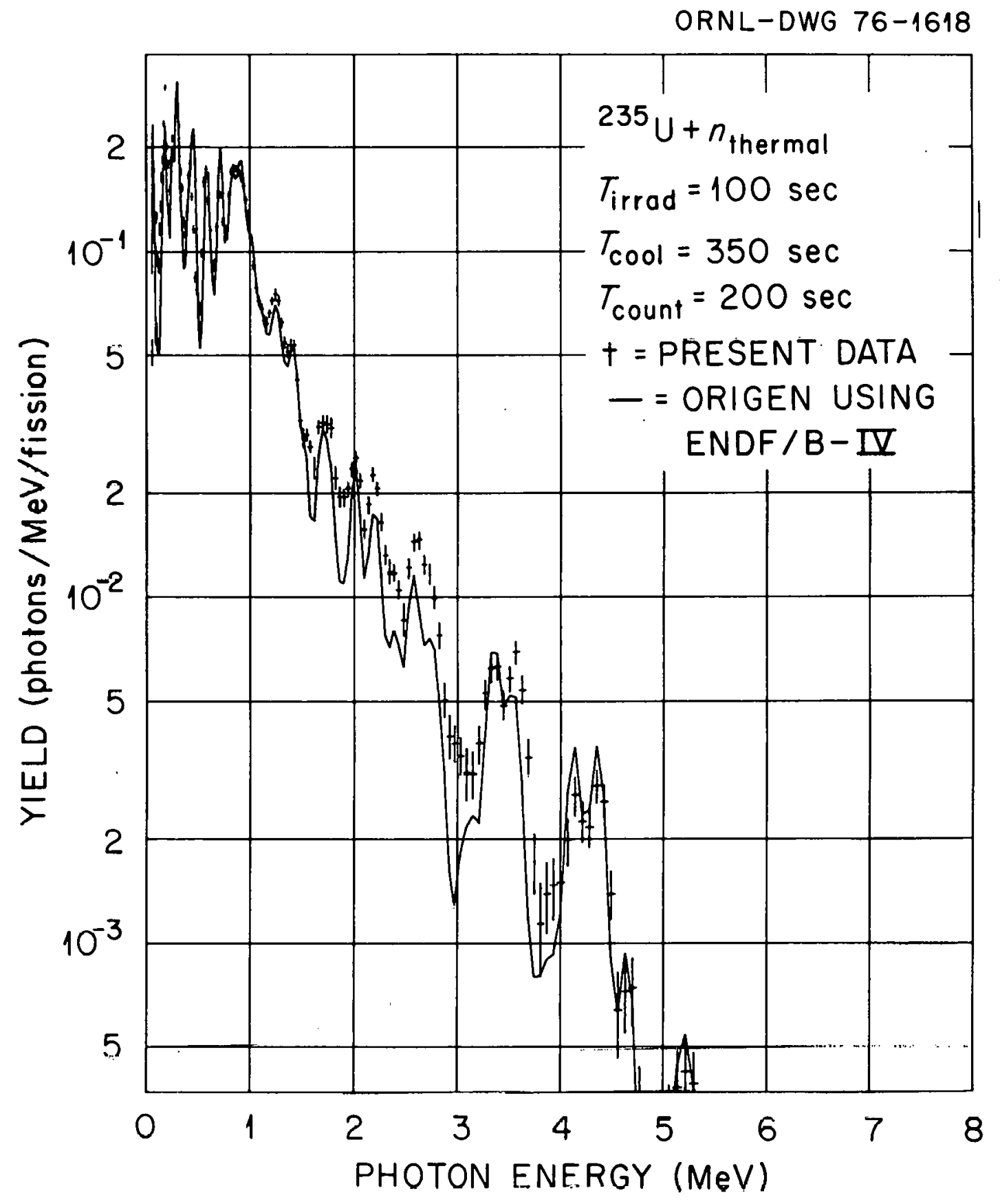

Fig. A-4. Comparison of Present Gamma-Ray Spectra (crosses) from thermal-neutron fission of $235 \mathrm{U}$ with calculated spectra (solid line) using ORIGEN. The irradiation time was $100 \mathrm{sec}$. The cooling time and counting interval is given in the legend. 
the normalization was made using the results of Weston et al. The neutron flux was measured using the ${ }^{10} B(n, \alpha)$ reaction; the energy variation used for this reaction was that given in ENDF/B-III.

The pulsed-neutron beam for these measurements was generated using the Oak Ridge Electron Linear Accelerator. A large liquid scintillator about $40 \mathrm{~m}$ from the neutron source was used to detect the prompt gamma-ray cascades resulting from neutron absorption in the sample. The time interval between the burst of neutrons and the detection of the absorption event was used to establish the neutron energy scale. The sample of the fissile isotopes was contained in multiplate (pulse) ionization chambers and those neutron absorption events detected in coincidence with a pulse from the ionization chamber were defined as fission events.

In general for ${ }^{239} \mathrm{Pu}$ and ${ }^{235} \mathrm{U}$, these experiments indicated lower neutron fission cross sections than contained in ENDF/B-III for energies above $10 \mathrm{keV}$. The measured values of the ratio $\alpha$, neutron capture-toneutron fission, for ${ }^{239} \mathrm{Pu}$ agree within errors with those derived from ENDF/B-III, Mat 1159. For the present measurements, the uncertainty on $\alpha$ for $23{ }^{9} \mathrm{Pu}$ is $211 \%$ at $10 \mathrm{keV}$ and increases to $230 \%$ at $100 \mathrm{keV}$.

The experimental results for the neutron capture cross section for ${ }^{197} \mathrm{Au}$ are $215 \%$ lower than the ENDF/B-III values. The measurements of the ratio of the neutron fission cross section for ${ }^{233} \mathrm{U}$ to that for ${ }^{235} \mathrm{U}$ are generally higher than the ENDF/B-III values by $25 \%$.

\section{e. Parameters of the Subthreshold Fission Structure in ${ }^{240} \mathrm{Pu}$ *, $* \star$ (George F. Auchampaught and Lawrence W. Weston)}

The neutron subthreshold fission cross section of $2{ }^{40} \mathrm{Pu}$ has been measured from $500 \mathrm{eV}$ to $10,000 \mathrm{eV}$ using the Oak Ridge Electron Linear Accelerator neutron facility. A total of 82 fission widths were obtained from area and shape analys is of those resonances which define the class II states at $\simeq 782 \mathrm{eV}, \simeq 1405 \mathrm{eV}, \cong 1935 \mathrm{eV}$, and $\cong 2700 \mathrm{eV}$. The average square of the coupling matrix element for the first three class II states is $1.85 \pm 1.43 \mathrm{eV}^{2}$ (S.D.). The average class II fission width is $2.5 \pm$ $1.0 \mathrm{eV}$ (S.D.). Approximately 22 clusters of class I resonances were observed below $10 \mathrm{keV}$, which results in a value of $450 \pm 50 \mathrm{eV}$ for the average class I level spacing. Assuming parabolic inner and outer barriers, the following barrier parameters were obtained:

$$
v_{A}-B_{n} / h \omega_{A}=0.71_{-0.09}^{+0.21} \text { and } v_{B}-B_{n} / h \omega_{B}=0.53 \begin{aligned}
& +0.09 \\
& -0.06
\end{aligned} \text {. }
$$

\footnotetext{
* Abstract of LA-UR 75-1318, LASL; also submitted for publication in Phys. Rev.

** Relevant to request Nos. 67130 and 72089-90.

+ Los Alamos Scientific Laboratory, Los Alamos, New Mexico 87545.
} 
f. The ${ }^{238} \mathrm{U}$ Subthreshold Neutron Induced Fission Cross Section

Subthreshold fission in the ${ }^{238} \mathrm{U}$ nucleus has been measured by Silbert and Bergen, ${ }^{1}$ Block et $a 1 .,^{2}$ and Blons. ${ }^{3}$ We report here recent high resolution measurements performed at the ORELA facility for neutron energies between $600 \mathrm{eV}$ and $2 \mathrm{MeV}$. The ORELA was operated at $800 \mathrm{pps}$ with neutron bursts $30 \mathrm{nsec}$ wide and a power of $40 \mathrm{~kW}$ on target. The resolution was $2 \mathrm{eV}$ at $600 \mathrm{eV}$ and $500 \mathrm{eV}$ at $100 \mathrm{keV}$. The detector was a fission chamber divided in two sections. The first section contained $4.5 \mathrm{~g}$ of ${ }^{238 \mathrm{U}}\left(2 \mathrm{ppm}\right.$ of $\left.{ }^{235 \mathrm{U}}\right)$ and the second section had $.65 \mathrm{~g}$ of highly enriched ${ }^{235} \mathrm{U}$.

The time-of-flight spectrum between $600 \mathrm{eV}$ and $100 \mathrm{keV}$ is shown in Fig. A-5. The data were reduced to fission cross sections by a ratio measurement to the ${ }^{235} \mathrm{U}$ count rate and the ENDF/B-IV evaluation of the ${ }^{235} \mathrm{U}$ fission cross section.

The average subthreshold fission cross section between 10 and $100 \mathrm{keV}$ was found to be $44 \pm 6 \mu \mathrm{b}$, which compares well with the values of $50 \pm 15 \mu \mathrm{b}$ and $41 \pm 16 \mu \mathrm{b}$ obtained by Silbert and Bergen ${ }^{1}$ and Block et al., ${ }^{2}$ respectively.

Between $600 \mathrm{eV}$ and $57 \mathrm{keV}, 28$ subthreshold fission clusters were clearly identified. The fission clusters at $721 \mathrm{eV}$ and $1.2 \mathrm{keV}$ were resolved into five and four resonances, respectively.

The present results have been interpreted on the basis of Strutinsky' ${ }^{4}$ double-humped fission barrier and the formalism of Weigmann ${ }^{5}$ and Lynn. ${ }^{6}$ The average level spacing for the Class II levels was $D_{I I}=1.8 \mathrm{keV}$. This yields $E_{I I}=1.8 \mathrm{MeV}$ for the height of the second minimum of the fission barrier above the ground state.

The fission areas for the two resolved clusters are in good agreement with the data of Block et al. ${ }^{2}$ A value of $1.4 \pm .3 \mathrm{meV}$ was found for the fission width of the $721-\mathrm{eV}$ resonance. For the unresolved fission clusters, the average fission width of the Class II levels was found to be $\left\langle\mathrm{I}_{\mathrm{f}}^{\mathrm{I}} \mathrm{I}_{\rangle}=.8 \pm .2 \mathrm{meV}\right.$. The distribution of the Class II fission widths was a $x^{2}$-distribution consistent with the presence of two open fission channels. From the present value of $\left\langle\Gamma_{f}^{I I}\right\rangle$, the Hill-Wheeler formula and

* Abstract of paper to be presented at ANS Meeting, Toronto, June 1976.

** Relevant to request Nos. 67203 and 69416 .

+ An IAEA fellow from Comission Nacional Energia Atomica, Argentina.

1 M. B. Silbert and D. W. Bergen, Phys. Rev. C 4, 220 (1971).

2 R. C. Block, et al., Phys. Rev. Letters, 31247 (1973).

3 J. Blons, C. Mazur and D. Paya, Conference on Neutron Cross Sections and Technology, NBS Special Publication 425 (1975) p. 642.

4 V. M. Strutinsky, Nucl. Phys. A95, 420 (1967).

5 H. Weigmann, Z. Phys. 214,7 (1968).

6 J. E. Lynn, AERE-R5891 (1968). 
ORNL-DWG 76-1703

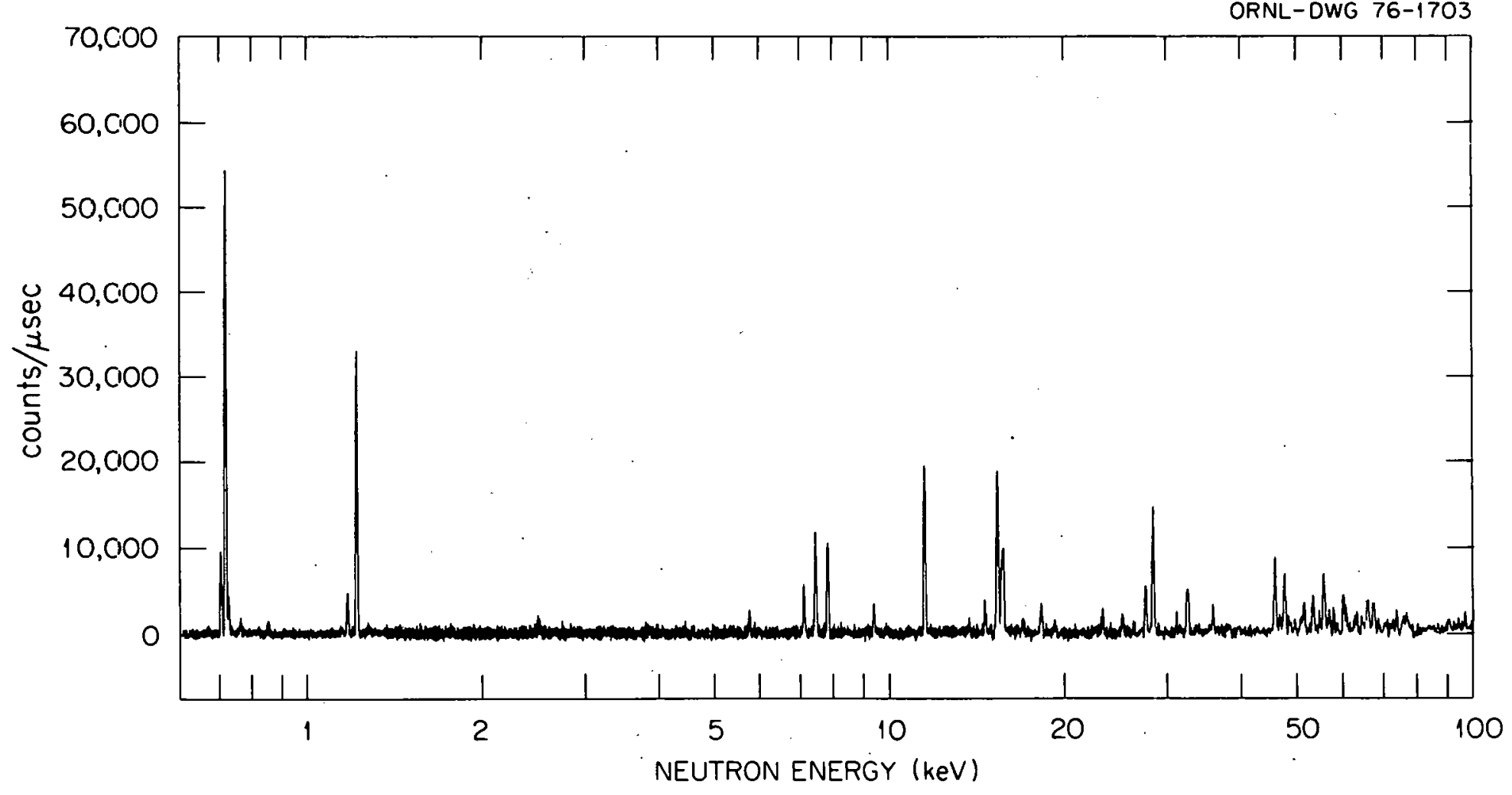

Fig. A-5. Time-of-Flight Spectrum Between $600 \mathrm{eV}$ and $100 \mathrm{keV}$. 
Specht's systematics for the lifetime of the shape fission isomers, one obtains a value of $6.3 \mathrm{MeV}$ for the height of the second barrier and a value of .7 MeV for the inverse curvature of the second barrier. Both values are in good agreement with the evaluation of Back et al. ${ }^{2}$

From the high energy data and the neutron binding energy in the ${ }^{238} \mathrm{U}$ nucleus, an upper bound of the height of the fission barrier was estimated at $6.3 \mathrm{MeV}$. This indicates that the height of the first barrier is either equal to or smaller than $6.3 \mathrm{MeV}$.

\section{h. High Resolution Measurement of the Neutron Induced ${ }^{238} \mathrm{U}$ Sub-

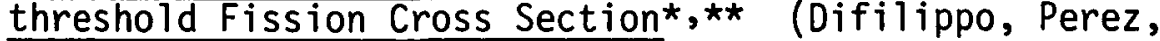 de Saussure, 0lsen and Ingle)}

Subthreshold fission in the ${ }^{298} \mathrm{U}$ nucleus has been measured by Silbert and Bergin, ${ }^{3}$ Block et al., ${ }^{4}$ and Blons. ${ }^{5}$ We report here recent high resolution measurements performed at the ORELA facility for neutron energies between $600 \mathrm{eV}$ and $1 \mathrm{MeV}$. The ORELA was operated at $800 \mathrm{pps}$ with neutron bursts $5 \mathrm{nsec}$ wide and a flight path of about $40 \mathrm{~m}$. The detector was a fission chamber divided in ten sections. The first eight sections contained a total of $4.5 \mathrm{~g}$ of ${ }^{238} \mathrm{U}(2 \mathrm{ppm}$ of $235 \mathrm{U})$ and the last two sections had a total of $.65 \mathrm{~g}$ of highly enriched $235 \mathrm{U}$.

The experimental resolution varied between 6 to $10 \mathrm{eV}$ in the region between $600 \mathrm{eV}$ and $10 \mathrm{keV}$, and was $100 \mathrm{eV}$ at $100 \mathrm{keV}$. The good resolution achieved in this experiment allows, in principle, resolving of the fission clusters below $20 \mathrm{keV}$, in terms of Class I levels. The analysis of the data up to $100 \mathrm{keV}$ indicates an average level spacing for the Class II levels of about $2 \mathrm{keV}$.

In a previous measurement, ${ }^{6}$ performed on a $20 \mathrm{~m}$ flight path at ORELA, a large fission cluster was observed around $150 \mathrm{keV}$, which was tentatively identified with a vibrational cluster. With the present resolution this cluster split into individual clusters with an average spacing of $4 \mathrm{keV}$, which is consistent with the expected spacing for a Class II, $J=3 / 2$, family of levels.

* Abstráct of paper to be presented at 1976 Int. Conf. Interactions of Neutrons with Nuclei, Lowe11, Massachusetts, July 6-9, 1976.

** Relevant to request Nos. 67203 and 69416 .

1 H. J. Specht, Physica Scripta 10A (1974).

2 B. B. Back et al., Third Conf. Physics and Chemistry of Fissinn, Rochester $(19 / 3)$, p. 8, IAEA-SM-174/201.

3 M. G. Silvert and D. W. Bergin, Phys. Rev. C 4, 220 (1971).

4 R. C. Block et al., Phys. Rev. Letters $31,24 \overline{7}(1973)$.

5 J. Blons et al., Proc. Conf. Nuclear Cross Sections and Technology, NBS Special Publication 425 (1975) p. 64?.

6 F. C. Difilippo et al., Trans. Am. Nucl. Soc., Toronto, June 1976, to be published. 
In the energy range between $200 \mathrm{keV}$ up to the first fission cross section plateau at $900 \mathrm{keV}$, several fission clusters have been observed. Their average spacing of around $50 \mathrm{keV}$ is too small for vibrational clusters. One tentatively concludes that we are observing clusters arising from the coupling of rotational bands and particle levels in the second well.

Similar structures were observed in the measurement of the subthreshold fission cross section of ${ }^{232} \mathrm{Th}$, by $\mathrm{Blons},{ }^{1}$ and have been discussed by Michaudon. ${ }^{2}$

\section{h. Neutron Absorption Cross Section of ${ }^{241} \mathrm{Am}{ }^{\star}, \star \star \quad$ (L. W. Weston and J. H. Todd)}

The ${ }^{241} \mathrm{Am}$ neutron absorption cross section, which is predominantly capture, has been measured from $0.01 \mathrm{eV}$ to $370 \mathrm{keV}$ neutron energy. The Oak Ridge Electron Linear Accelerator (ORELA) was used as the source of pulsed neutrons. Resonance parameters (SLBW) have been derived for the data up to $50 \mathrm{eV}$. The capture gamma-ray detector used was the "total energy detector," which is a modification of the Moxon-Rae detector. This detector required that the events be weighted by their pulse height in the detector and that the net efficiency of the detector be low. The cross section was normalized at thermal neutron energies ( 0.02 to $0.03 \mathrm{eV})$ and the shape of the neutron flux was measured relative to the ${ }^{10} \mathrm{~B}(n, \alpha)$ cross section up to $2 \mathrm{keV}$ and relative to the ${ }^{6} \mathrm{Li}(n, \alpha)$ cross section at higher neutron energies. The results of the measurement indicate a lower cross section ( $25 \%$ ) between 0.3 and $100 \mathrm{eV}$ than has been previously indicated and an appreciably higher cross section (by $100 \%$ at $100 \mathrm{keV}$ ) from 20 to $370 \mathrm{keV}$.

Figures $A-6$ and A-7 give the results compared to ENDF/B-IV.

i. The Neutron Total Cross Section and Resonance Parameters of ${ }^{249} \mathrm{Bk}+$ (J. A. Harvey, R. W. Benjamin,中 N.W. Hill and S. Raman)

Several years ago a cooperative program was initiated between ORNL and the Savannah River Laboratory to measure the neutron total cross section of the heavy actinides as samples became available. Initially the interest in the cross sections of the transplutonium isotopes was to enable more accurate calculations of ${ }^{252} \mathrm{Cf}$ production. Recently, however,

* Submitted for publication in Nucl. Sci. Eng.

** Relevant to request Nos. 67135-36, 70048, 72099 and 74127.

+ Kelevant to request No. 67151 .

\# Savannah River Laboratory, Aiken, S.C.

1 J. Blons et al., Proc. Conf. Nuclear Cross Sections and Technology, NBS Special Publication 425 (1975) p. 642.

2 A. Michaudon, ibid., p. 202. 


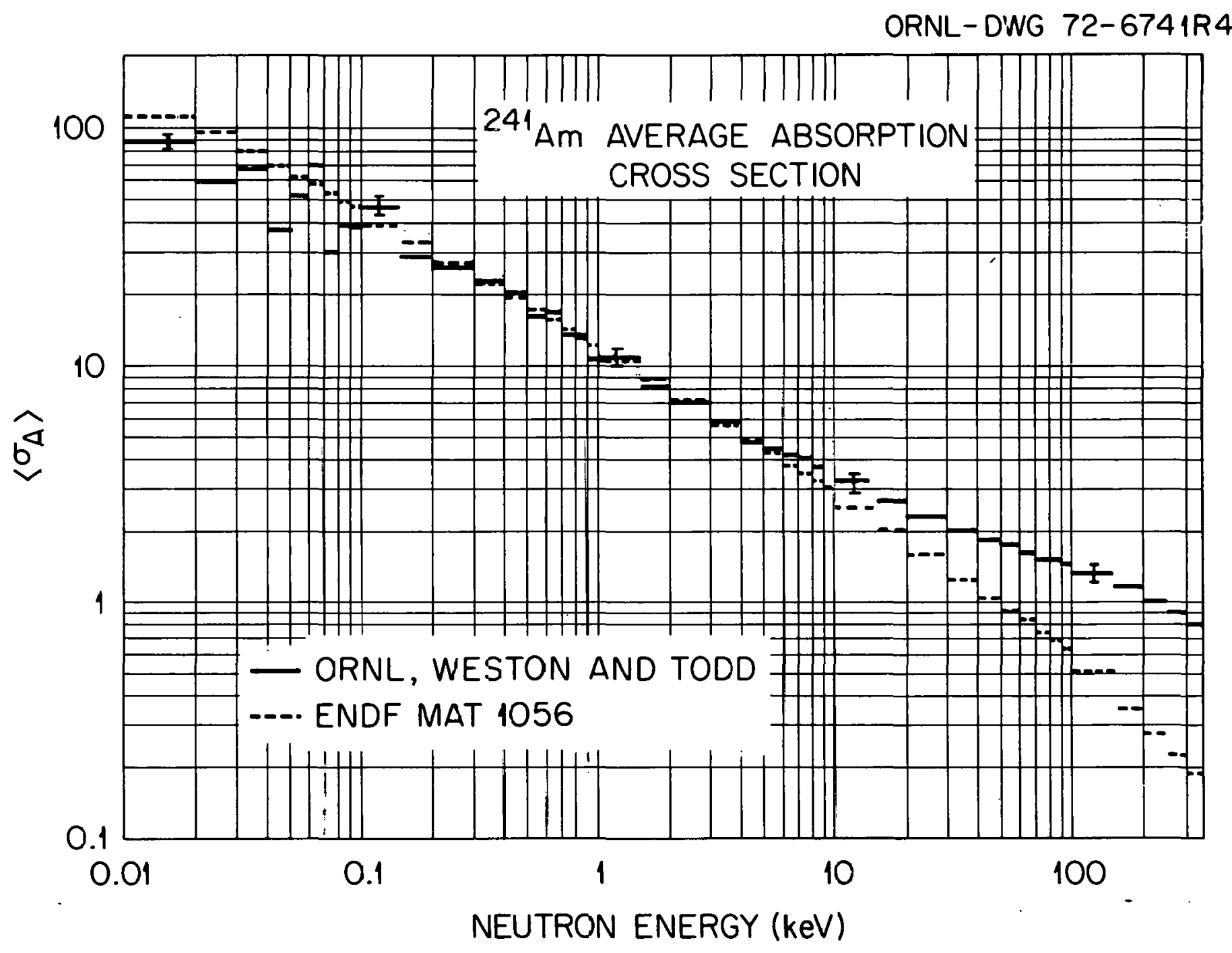

Fig. A-6. Comparison with ENDF/B-IV. 


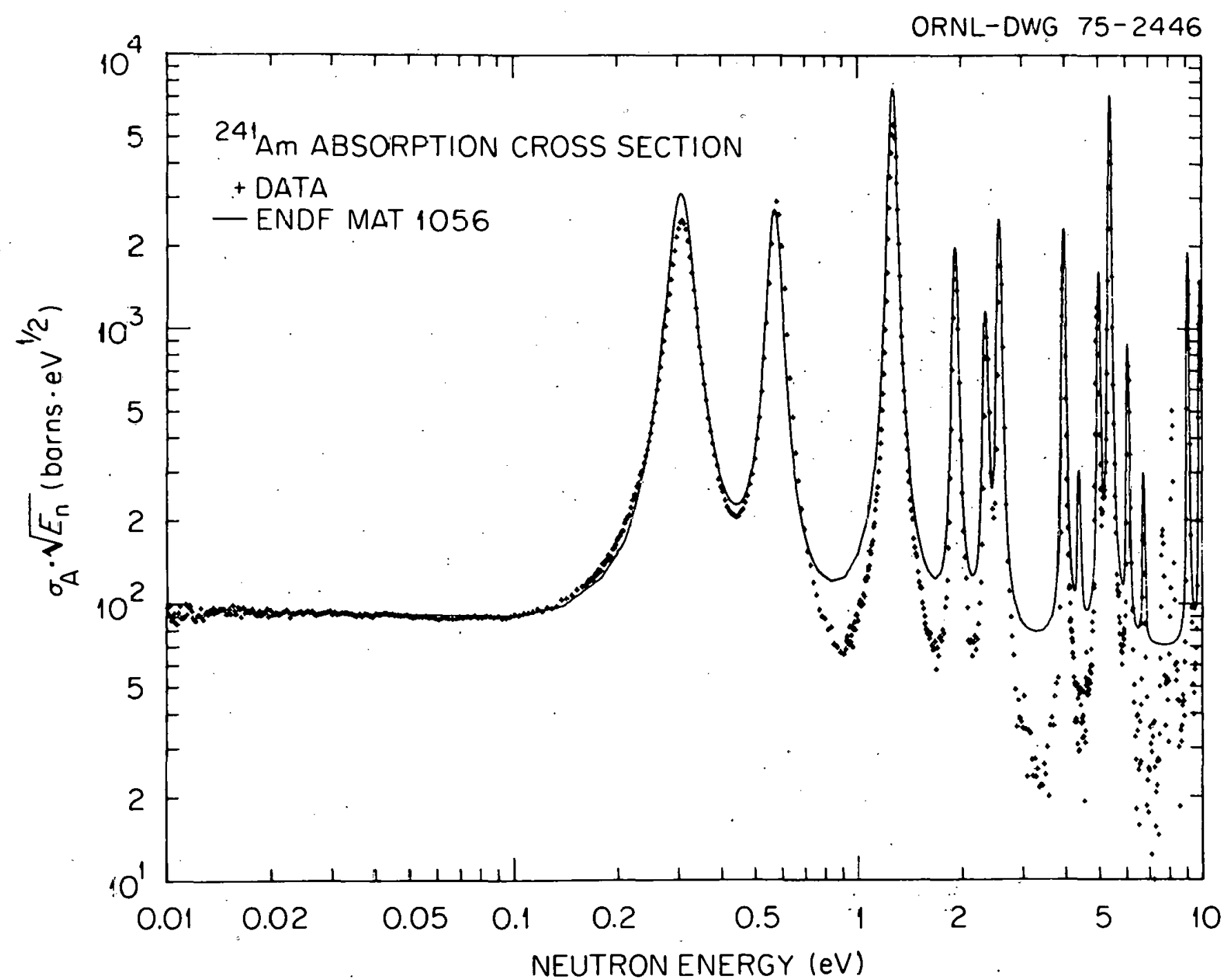

Fig. A-7. Comparison with ENDF/B-IV. 
the study of the problem of managing radioactive wastes, particularly the long-lived actinides, and the possibility of recycling these long-lived actinides has emphasized the need for additional and better data on many heavy isotopes. Neutron total cross section measurements upon small samples (too small and/or radioactive to permit a direct capture cross section measurement) can readily be interpreted to yield the absorption cross section in the thermal energy range and often up to $2100 \mathrm{eV}$.

This year a 6 -mg sample of ${ }^{249} \mathrm{Bk}$ became available through the cooperation of John Bigelow of the TRU facility. Two samples were prepared from this material, a "thick" sample containing $5.3 \mathrm{mg}(\mathrm{N}=$ 0.00061 atoms/barn) and a "thin" one containing $20.8 \mathrm{mg}$. The sample holders had inside diametcrs of only 1.6 IIII and the neutron beam was collimated to a diameter of $1.3 \mathrm{~mm}$. The samples wore cooled with liquid nitrogen to reduce the Doppler brodeniny which is greater than the neutron energy resolution up to $\sim 100 \mathrm{eV}$. Measurements were made upon the samples using a $11.0-\mathrm{cm}$ diameter, $1.3-\mathrm{cm}$ thick ${ }^{6} \mathrm{Li}-\mathrm{glass}$ scintillator located at a 17.87 meter flight path. The measurements covered the energy range from 0.005 to $210000 \mathrm{eV}$ with an energy resolution of $0.3 \%$.

A total of 47 resonances below $\sim 130 \mathrm{eV}$ were observed. A large resonance was observed at $0.197 \mathrm{eV}$ which is responsible for most of the thermal absorption cross section, which departs markedly from a $1 / v$ energy dependence. The average level spacing based on the resonances up to $20 \mathrm{eV}$ is $1.1 \mathrm{eV}$. The large resonance in ${ }^{249} \mathrm{Cf}$ at $0.70 \mathrm{eV}$ was also observed even though there was only $\sim 2 \%{ }^{249} \mathrm{Cf}$ in the sample (i.e., 210 $\mu \mathrm{gm})$ at the time of measurement. Additional measurements will be made after about half of the 330 -day ${ }^{249} \mathrm{BK}$ has decayed into ${ }^{249} \mathrm{Cf}$ in order to obtain parameters for the resonances in ${ }^{249} \mathrm{Cf}$ as well as its thermal absorption cross section. These measurements will be combined with the fission cross section measurements of Dabbs et. al. ${ }^{1}$ to obtain a complete set of jardilleters of the low energy resonances of $249 \mathrm{C} . \mathrm{f}$.

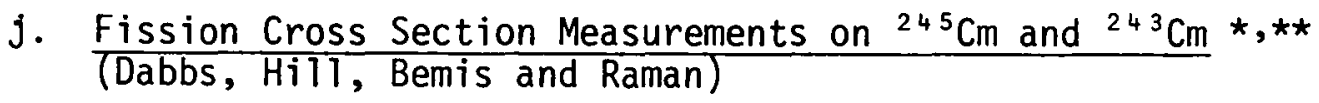

Fission cross-section determinations are in progress with small ultra-pure and highly alpha active actinide samples using a new type of hemispherical plate ionization chamber and fast current sensitive amplifiers. ${ }^{2} 10 \mu \mathrm{g}$ samples are adequate for such measurement at a $10 \mathrm{~m} \mathrm{flight}$ path at ORELA, using irradiations of several hundred hours and power levels above $40 \mathrm{~kW}$. The basic resolution is about $4 \mathrm{~ns} / \mathrm{m}$. In the case

* Abstract of paper to be presented at 1976 Int. Conf. Interactions of Neutrons with Nuclei, Lowe11, Massachusetts, July 6-9, 1976.

** Relevant to request No. 67145 .

1 "Neutron-Induced Fission of $248 \mathrm{Cf}, " \mathrm{~J}$. W. Dabbs et al., Physics Division Annual Report, ORNL-4937, May 1974, p. 181.

2 J. W. T. Dabbs et al., Proc. Conf. Nuclear Cross Sections and Technology, NBS Special Publication 425 (1975) p. 81. 
of ${ }^{243} \mathrm{Cm}$ a clear determination of a spontaneous fission rate of 0.01 counts/sec in the presence of an alpha particle emission rate of $10^{7} / \mathrm{sec}$ has been demonstrated, with $>95 \%$ efficiency for the fission counts. Preliminary results showing a number of new resonances at low energies will be presented. The importance of such measurements to the nuclear waste question will be discussed briefly.

k. Measurement of Neutron Transmissions from $0.52 \mathrm{eV}$ to $4.0 \mathrm{keV}$

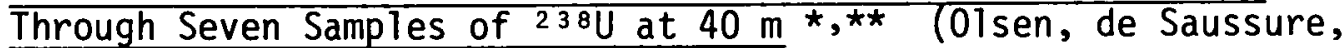
Perez, Silver, Ingle and Weaver)

Neutron transmission through 1.5-, 5-, 10-, 30-, 100-, 425-, and 1425-mil samples of depleted ${ }^{238} \mathrm{U}$ were measured from $0.52 \mathrm{eV}$ to $4.0 \mathrm{keV}$ using the ORELA pulsed electron linac neutron source and time-of-flight technique with a $1-\mathrm{mm}^{6} \mathrm{Li}-\mathrm{glass}$ detector with a $\mathrm{flight}$ path of $40 \mathrm{~m}$. The measurements are tabulated and compared with transmission calculated from the ENDF/B-IV total cross section. In addition, the 1425-mil transmission from 50 to $300 \mathrm{eV}$ is compared with transmissions calculated by using multilevel formalisms, and some neutron widths are extracted with area analysis and compared with those from previous measurements.

८. Resonance Parameters of the 6.67-, 20.9-, and 36.8-eV Levels in $238 \mathrm{U}+, \star \star$ (01sen, de Saussure, Perez and Difilippo)

The ENDF/B-IV ${ }^{238} \mathrm{U}$ cross sections (MAT-1262) yield an effective capture resonance integral in strongly self-shielded situations which is too high. ${ }^{1}$ This situation suggests that the ENDF/B capture widths for the first few $s$-wave levels may be too large. Recent ORELA measurements ${ }^{2}$ of transmission through ${ }^{238} \mathrm{U}$ have been analyzed with a multilevel formula to determine the parameters of the 6.67-, 20.9-, and 36.8-eV levels. These three levels provide $86 \%$ of the infinitely dilute capture resonance integral.

* Abstract of ORNL/TM-5256 (March 1976).

** Relevant to request Nos. 69286-89.

+ Abstract of paper to be presented at ANS Meeting, Toronto, June 1976.

1 An extended discussion on this problem is contained in the various papers of Seminar on ${ }^{238} \mathrm{U}$ Resonance Capture, Brookhaven National Laboratory, BNL-NCS-50451, ed., S. Pearlstein, 1975.

2 D. K. Olsen, G. de Saussure, E. G. Silver, and R. B. Perez, Seminar on $2{ }^{38} \mathrm{U}$ Resonance Capture, Brookhaven National Laboratory, BNL-NCS-50451, p. 95, ed., S. Pearlstein, 1975; D. K. 01sen, G. de Saussure, E. G. Silver, and R. B. Perez, Trans. Am. Nucl. Soc. 21, 505 (1975); and D. K. Olsen, G. de Saussure, R. B. Perez, E. G. Silver, R. W. Ingle, and $H$. Weaver, "Measurement of Neutron Transmission from $0.52 \mathrm{eV}$ to $4.0 \mathrm{keV}$ Through Seven Samples of ${ }^{238} \mathrm{U}$ at $40 \mathrm{~m}, "$ ORNL/TM-5256, January 1976. 
The data consist of transmissions through $3.62-, 1.08-, 0.254-$, $0.0762-, 0.0254-, 0.0127-$, and $0.0036-\mathrm{cm}$ metal samples. These seven transmissions were simultaneously analyzed over many resonances with the least-squares computer code $\mathrm{SIOB}^{1}$ which contains Gaussian resolution and Doppler broadening and employs a multilevel Breit-Wigner cross section formalism ${ }^{2}$ with "picket fences" terms to account for distant levels, both bound and unbound. In addition to the resonance parameters, the code allows the effective radius, normalizations, backgrounds, and picket fence terms to be fitted parameters.

An effective radius of $.949 \times 10^{-12} \mathrm{~cm}$ was obtained, independent of the details of the bound levels, by fitting the transmission data from 55 to $500 \mathrm{eV}$. The cross section of the energy region of interest from 0.52 to $55.0 \mathrm{eV}$ is sensitive to the bound levels. Both the same effertive radius of $948 \times 10^{-12} \mathrm{~cm}$ and a minimum in $x^{2}$ was obtained for this lowest energy region with a picket fence of bound levels extending from $-80 \mathrm{eV}$ to $-\infty$. Fig. A-8 shows this fit.

Table A- 1 compares the neutron and radiation widths from this fit with those contained in ENDF/B-IV. The errors immediately following these widths are the statistical standard deviations from the fit. The numbers in parentheses are deviations corresponding to the uncertainty in the effective Doppler temperature, i.e. $300^{\circ} \pm 5^{\circ} \mathrm{K}$. The statistical standard deviations for the widths are small. However, the widths depend strongly on the cross-section model and parameters, and the statistical errors contain no estimate of the systematic errors in the data. The uncertainties associated with systematic errors in the data are estimated to be an order of magnitude larger than the statistical errors quoted in Table $A-1$. It is concluded that radiation widths smaller than those contained in ENDF/B-IV are required to reproduce these transmission data. These smaller capture widths would significantly reduce the discrepancies between calculated and measured capture resonance integrals, ${ }^{3}$

TABLE A-1. Neutron and Radiation Widths

\begin{tabular}{cccccc}
\hline & \multicolumn{2}{c}{ LEAST-SQUARES FIT } & \multicolumn{2}{c}{ ENDF/B-IV } \\
\hline $\mathrm{E}(\mathrm{eV})$ & \multicolumn{1}{c}{$\Gamma_{\mathrm{n}}(\mathrm{meV})$} & $\Gamma_{\gamma}(\mathrm{meV})$ & $\Gamma_{n}(\mathrm{meV})$ & $\Gamma_{\gamma}(\mathrm{meV})$ \\
$6.6 /$ & $1.482 \pm .002( \pm .006)$ & $22.96 \pm .04( \pm .02)$ & 1.50 & 25.6 \\
20.9 & $10.19 \pm .01( \pm .03)$ & $22.46 \pm .04( \pm .02)$ & 8.80 & 26.8 \\
36.8 & $33.05 \pm .03( \pm .05)$ & $22.29 \pm .03( \pm .02)$ & 31.1 & 26.0 \\
\hline
\end{tabular}

I $G$ de Saussure, D. K. 01 sen, and R. B. Perez, to be published.

${ }^{2}$ H. A. Bethe, Rev. Mod. Phys. 9, 69 (1937); also H. A. Bethe and G. P1.aczek, Phys. Rev. 51, 450 (1939).

3 M. R. Bhat, Seminar on ${ }^{2}{ }^{3} \mathrm{U}$ capture, Brookhaven National Laboratory, BNL-NCS-50451, p. 244, ed., S. Pearlstein, 1975. 


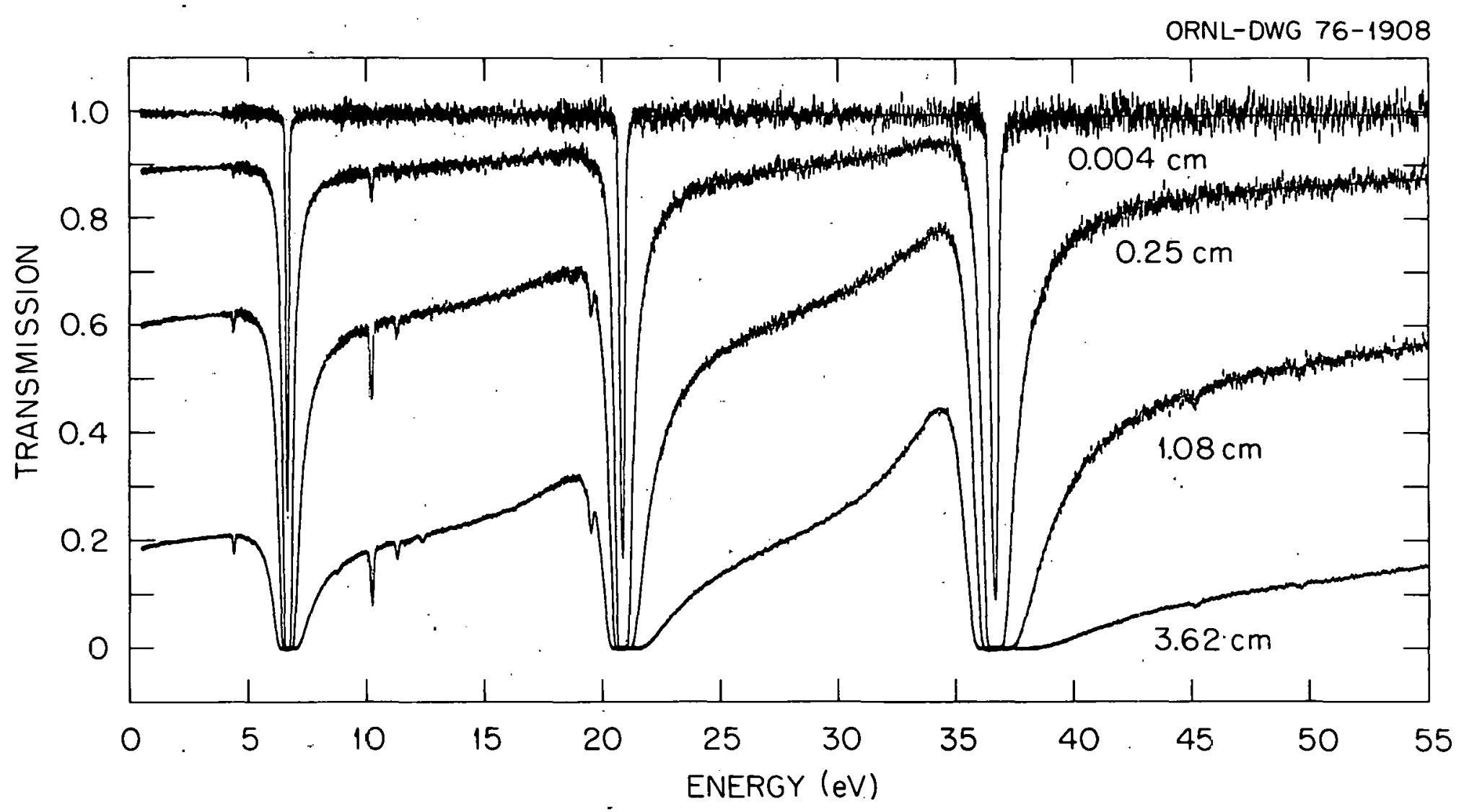

Fig. A-8. Simultaneous Least-Squares fit with the Multilevel Computer Code, SIOE, to neutron transmission from 0.52 to $55.0 \mathrm{eV}$ through $3.62-, 1.08-, 0.254-$, $0.0762-, 0.0254-, 0.0127-$, and $0.0035-\mathrm{cm}$ samples of ${ }^{238} \mathrm{U}$. For clarity the fit to the data from the $0.0762-, 0.0254-$, and $0.0127-\mathrm{cm}$ samples is not shown. 
m. Three papers were presented at the IAEA Advisory Group Meeting on Transactinium Isotope Nuclear Data at Karlsruhe, 1975, and will be published in the proceedings:

General Survey of Applications Which Require Actinide Nuclear Data (S. Raman)

This review paper discussed the actinide waste problem, the buildup of toxic isotopes in the fuel, the neutron activity associated with irradiated fuel, the ${ }^{252} \mathrm{Cf}$ buildup problem, and the production of radioisotope power sources as broad areas that require actinide cross-section data. Decay data enter into the area of radiological safety and health physics. This paper also discussed a few cross-section measurements in progress at the Oak Ridge Electron Linear Accelerator. The availability of actinide samples through the Transuranium Program at 0ak Ridge is discussed in considerable detail. The present data status with respect to the various applications is reviewed along with recommendations for improving the data base.

Some Activities in the United States Concerning the Physics Aspects of Actinide Waste Recycling (S. Raman)

This review paper briefly discussed the reactor types being considered in the United States for the purpose of actinide waste recycling. The reactor types include thermal reactors operating on the $3.3 \%{ }^{23} \mathrm{U}-{ }^{2} 32 \mathrm{U}$ and the ${ }^{233} \mathrm{U}-{ }^{232}$ Th fuel cycles, liquid metal fast breeder reactors, reactors fueled entirely by actinide wastes, gaseous fuel reactors and fusion reactors. This paper also discusses cross section measurements in progress or planned toward providing basic data for testing the recycle concept.

Neutron Capture Cross Sections of the Actinides (L. W. Weston)

\section{Integral Measurements}

a. Measurement of Secondary Neutrons and Gamma Rays Produced by Neutron Interactions with Nitrogen and Oxygen over the Incident Energy Range 1 to $20 \mathrm{MeV}^{\star}$ (G. L. Morgan)

The spectra of secondary neutrons and gamma rays produced by neutron interaction in thick samples $(\approx 1$ mean free path) of liquid nitrogen and liquid oxygen have been measured as a function of the incident neutron energy over the range 1 to $20 \mathrm{MeV}$. Data were taken for angles of $30^{\circ}, 55^{\circ}, 90^{\circ}$, and $125^{\circ}$. A linac (ORELA) was used as a neutron source with a 47-m flight path. Incident energy was determined by time-of-flight,

* Abstract of ORNL-TM-5023 (October 1975). 
while secondary spectra were determined by pulse-height unfolding techniques. The results of the measurements are presented in forms suitable for comparison to calculations based on the evaluated data files.

b. Measurement of Secondary Neutrons and Gamma Rays Produced by Neutron Interactions in Aluminum over the Incident Energy Range 1 to $20 \mathrm{MeV}$ * (G. L. Morgan)

The spectra of secondary neutrons and gamma rays produced by neutron interaction in a thin sample $(\approx 1 / 6$ mean free path) of aluminum have been measured as a function of the incident neutron energy over the range 1 to $20 \mathrm{MeV}$. Data were taken at an angle of $125^{\circ}$. A 1 inac (ORELA) was used as a neutron source with a 47-m flight path. Incident energy was determined by time-of-flight, while secondary spectra were determined by pulse-height unfolding techniques. The results of the measurements are presented in forms suitable for comparison to calculations based on the evaluated data files.

c. Measurement of Secondary Neutrons and Gamma Rays Produced by Neutron Interactions in Silicon Dioxide over the Incident Energy Range 1 to $20 \mathrm{MeV}$ ** (G. L. Morgan)

The spectra of secondary neutrons and gamma rays produced by neutron interactions in a thick ( $\approx 1$ mean free path) sample of silicon dioxide have been measured as a function of the incident neutron energy over the range 1 to $20 \mathrm{MeV}$. Data were taken at an angle of $90^{\circ}$. A linac (ORELA) was used as a neutron source with a 47-m flight path. Incident energy was determined by time-of-flight, while secondary spectra were determined by pulse-height unfolding techniques. The results of the measurement are presented in forms suitable for comparison to calculations based on the evaluated neutron data files.

d. Measurement of Secondary Neutrons and Gamma Rays Produced by Neutron Bombardment of Water over the Incident Energy Range 1 to $20 \mathrm{MeV}+$ (G. L. Morgan)

The spectra of secondary neutrons and gamma rays pruduced by neutron bombardment of a thick ( $\approx 1$ mean free path) sample of water have been measured as a function of the incident neutron energy over the range 1 to $20 \mathrm{MeV}$. Data were taken for angles of $90^{\circ}$ and $140^{\circ}$. A 1 inac (ORELA) was used as a neutron source with a $47-\mathrm{m}$ flight path. Incident energy was determined by time-of-flight, while secondary spectra were determined by pulse-height unfolding techniques. The results of the measurements are presented in forms suitable for comparison to calculations based on the evaluated data files.

\footnotetext{
* Abstract of ORNL-TM-5072 (November 1975).

** Abstract of ORNL-TM-5024 (September 1975).

+ Abstract of ORNL-TM-5018 (August 1975).
} 
8. Experimental Techniques

a. Monte Carlo Calculations of Multiple-Pulse Pileup (J.W.T.J abbs)

In fission cross-section measurements, it is often desired to make measurements in the presence of an intense alpha particle background. It is obvious that with sufficiently fast electronic equipment the alpha pulse pileup can be reduced to unimportance but quantitative knowledge of the problem is needed to choose the allowable amounts of material with particular ionization chambers, gas scintillator systems, or segmented ionization chambers.

A new method for Montc Carlo calculdtions, which is applicable to pulse pileup to any desired multiplicity of nileup, has bcen developed and used with a minicomputer for pileups with as many as 20 pulses. Previously, closed-form calculations have been presented only for four pulses, except in the trivial case of square pulses. The present calculations are easily adaptable to unusual geometries such as in the hemispherical-plate fission chamber work described elsewhere in this report.

The method consists of ectting up a table of 32 values (nominally one value per nanosecond) for the pulse shape in question. A double interval in time, 64 units long, is established. Then, for each multiplicity $k$ (from 1 to some upper limit, say, 20) of pulses in the interval, $2 \mathrm{k}$ such pulses are distributed, to begin randomly in time over the double interval, and their amplitudes are added in each unit of the second half of the double interval, thus generating 32 values of pulse height for that $k$. After 250 repetitions of this process for each $k$ value, one has an 8000-member height distribution for that $k$. The pulse-height distributions are then weighted by the Poisson distribution factor, $p_{k}$, for the particular counting rate involved and are summed to qive the final pulse-height distribution. Cumparison with observed values in a practical case has established the validity of the calculation. Such calculations have been used in designing three experiments so far.

b. An Experimental System for Providing Data to Test Evaluated Secondary Neutron and Gamma-Ray-Production ) ross Sections over the Incident Neutron Energy Range from 1 to $20 \mathrm{MeV}$ *

(G. L. Morgan, T. A. Love and F. G. Perey)

A system is described which allows simultaneous measurement of secondary neutron and gamma-ray-production cross sections. Measurements can be made rapldly over wide energy ranges. An electron linac is used as a neutron source. Annular scattering samples located $47 \mathrm{~m}$ from the neutron source are viewed by a NE-213 scintillation counter. Multiparameter data acquisition is / one by on-line computer for incident neutron energies from 1 to $20 \mathrm{MeV}$.

* Nuc1. Intrum. Methods $\underline{128}, 125$ (1975). 


\section{B. DATA ANALYSES}

Most of the data procurement activities reported in the previous section were mainly motivated by a need in some application. Much work remains to be done after some data have been obtained before they become useful to applied users. In this section we report on some of these activities bearing directly upon this work: theoretical calculations, compilations, evaluations, validation of evaluated data, and sensitivity studies aimed at establishing adequacy of the data.

1. Theoretical Calculations

a. Calculated Nucleon Spectra at Several Angles from 192-, 500-, 700-, and 900-MeV Carbon-12 on Iron-56 * (H. W. Bertini, R. T. Santoro and 0.W. Hermann)

Neutron spectra were calculated as a function of angle between 0 and $110^{\circ}$ for ${ }^{12} \mathrm{C}$ on ${ }^{56} \mathrm{Fe}$ at 192,500,700, and $900 \mathrm{MeV}$. Proton spectra were calculated for the same angular range but for only $192-\mathrm{MeV}{ }^{12} \mathrm{C}$ on ${ }^{56} \mathrm{Fe}$. The most significant property of these spectra is that there is an appreciable number of neutrons emitted with energies greater than the incident energy per nucleon at all angles investigated.

b. Calculated Neutron Cross Sections for $\mathrm{Cu}$ and $\mathrm{Nb}$ up to $32 \mathrm{MeV}$ for Neutron Damage Analysis** (C. Y. Fu and F. G. Perey)

Cross sections for neutron interaction with $\mathrm{Cu}$ and $\mathrm{Nb}$, with emphasis on spectra of light particles from binary reactions, are calculated for neutron energies from 4 to $32 \mathrm{MeV}$ for estimating recoil probability densities for the analysis of damage experiments with a $\operatorname{Be}(d, n)$ neutron source. Nuclear model parameters were adjusted to reproduce the available cross-section data around $14 \mathrm{MeV}$. Helium production cross sections were also calculated for ${ }^{63} \mathrm{Cu}$ for neutrons below $20 \mathrm{MeV}$, as an illustration of the Hauser-Feshbach method for calculating tertiary reaction cross sections.

* Abstract of ORNL/TM-5161 (February 1976); also submitted for publication in Phys. Rev. C.

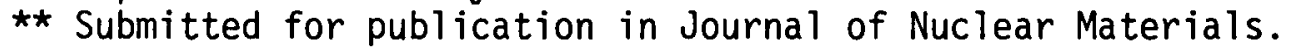




\section{ENDF/B Related Evaluations}

a. An Evaluation of Neutron and Gamma-Ray-Production Cross-Section Data for Lead* (C. Y. Fu and F. G. Perey)

A survey was made of the available information on neutron and gamma-ray-production cross-section measurements of lead. From these and from relevant nuclear-structure information on the $\mathrm{Pb}$ isotopes, we prepared recommended neutron cross-section data sets for lead covering the neutron energy range from $0.00001 \mathrm{eV}$ to $20.0 \mathrm{MeV}$. The cross sections are derived from experimental results available to February 1972 and from calculations based on optical-model, DWBA, and Hauser-Feshbach theories. Comparisons which show good agreement between theoretical and experimental values are displayed in a number of graphs. Also presented graphically are smonthed total rrosss sections, Legendre coefficients for angular dis tributions, and a representative energy distribution of gamma rays from resonance capture.

b. Consistent Calculations of Neutron and Gamma-Ray-Production Cross Sections for Ca-40 from 1 to $20 \mathrm{MeV}$ (C. Y. Fu)

Cross sections of neutron interaction with ${ }^{40} \mathrm{Ca}$ and the subsequent production of gamma rays are calculated and compared with experiments. Various nuclear models are judiciously applied for the calculation. The Hauser-Feshbach theory for binary reactions is extended to include tertiary reactions, which are important for ${ }^{40} \mathrm{Ca}$ from 10 to 20 $\mathrm{MeV}$. Continuum-level spins and parities are included in the gamma-ray production calculation to conserve angular momentum. An extensive measurement of gamma-ray production cross sections, available after all model parameters were fixed, is used to test the predictability of the models, particularly in the high energy range, where tertiary reactions contribute significantly.

c. Note on the ENDF/B-IV Representation of the ${ }^{238} \mathrm{U}$ Total Cross Section in the Resolved Resonance Energy Region ${ }^{\star \star}$ (de Saussure 01 sen and Perez)

The ENDF/B-IV prescription fails to represent correctly the ${ }^{238} \mathrm{U}$ total (and scattering) cross section between the levels of the resolved range. We show how this representation can be improved by properly accounting for the contribution of levels outside the resolved reqion to the cross section at energies inside the resolved region, and by substituting the morc precise multilevel Breit-Wigner furmula for the presently used single level formula. We illustrate the importance of computing accurately the minima in the total cross section by comparing values of the self-shielded capture resonance integral computed with ENDF/B-IV and with a more accurate cross-section model.

* Atomic Data and Nuclear Data Tables 16, 409 (1975).

** Submitted for publication in Nuclear Science and Engineering. 


\section{d. SUR, A Program to Generate Error Covariance Files* \\ (F. C. Difilippo)}

Covariance matrices were calculated for the ${ }^{238} \mathrm{U},{ }^{241} \mathrm{Pu}$, and ${ }^{239} \mathrm{Pu}$ fission cross sections and for the ${ }^{238} \mathrm{U},{ }^{240} \mathrm{Pu},{ }^{241} \mathrm{Pu}$, and ${ }^{239} \mathrm{Pu}$ capture cross sections. A computer program was written which uses the evaluated ENDF/B data files and the measured or evaluated (from other evaluations) cross sections for the calculation of the uncertainty files. An effort has been made to make the output of the program consistent with the ENDF/B error files format. A user's manual for the present code and references utilized in the covariance matrix calculations are given.

e. The Energy Dependence of the Neutron Absorption and Fission Cross Sections of ${ }^{235} \mathrm{U}$ and ${ }^{239} \mathrm{Pu}$ Below $1 \mathrm{eV}$ and the Wescott gFactors** (R. Gwin)

The energy dependence of recently published ${ }^{1}$ neutron absorption and fission cross sections $\left[\sigma_{a}(E)\right.$ and $\left.\sigma_{f}(E)\right]$ of ${ }^{235} \mathrm{U}$ and ${ }^{239} \mathrm{Pu}$ has been reexamined. The published data are normalized to ENDF/B-III values of $\sigma_{f}$ and $\sigma_{a}$. This paper compares the data of Ref. 1 to the recently released ENDF/B-IV values $\sigma_{f}$ and $\sigma_{a}$ for ${ }^{235} \mathrm{U}$ and ${ }^{239} \mathrm{Pu}$. Wescott $\mathrm{g}$ factors are calculated for fission $\left(g_{f}\right)$ and for absorption $\left(g_{a}\right)$.

f. A New Perturbation Formalism for the Complex Widths and Poles of the Transition T-Matrixt (Perez, de Saussure and 01sen)

form

The T-matrix of nuclear reaction theory can be written in the

$$
T_{c c^{\prime}}=i \sum_{v} \frac{g_{v c} g_{v c^{\prime}}}{\varepsilon_{v}-E},
$$

where $g_{v c}$ is the complex width for channel $c$ and $\varepsilon_{v}$ the complex poles of the transition T-matrix. We have shown that, in terms of a parameter $\tau(0<\tau \leq 1)$, the complex widths and poles of the T-matrix are obtained via the solution of two coupled Volterra equations.

In the present formalism the interaction contains both changes in the hamiltonian operator and the boundary conditions. The convergence of the method depends on the ratio of the elements of the interaction matrix

* Abstract of ORNL/TM-5223 (March 1976).

** Submitted for publication in Nuclear Science and Engineering.

+ Abstract of paper to be presented at 1976 Int. Conf. Interactions of Neutrons with Nuclei, Lowe11, Massachusetts, July 6-9, 1976.

1 R. Gwin, et al., "Measurement of the Neutron Capture and Fission Cross Sections of ${ }^{2}{ }^{9} \mathrm{Pu}$ and ${ }^{235} \mathrm{U}, 0.02 \mathrm{eV}$ to $200 \mathrm{keV}$, the Neutron Capture Cross Sections of ${ }^{197} \mathrm{Au}, 10$ to $50 \mathrm{keV}$, and Neutron Fission Cross Sections of ${ }^{235} \mathrm{U}, 5$ to $200 \mathrm{keV}, "$ Nucl. Sci. Eng. 59, 79 (1976). 
to the spacing of the complex poles rather than on the same ratio expressed in terms of the spacing of the R-matrix poles on the real axis, as it is the case in the usual perturbation approach.

The present method has been applied to neutron cross section calculations in cases of large level interference and to the study of intermediate structure phenomena.

\section{Validation of ENDF/B Evaluations Through Integral Measurements \\ a. Critical Experiments and the $2200 \mathrm{~m} / \mathrm{sec}$ Neutron Parameters* (R. Gwin)}

The use of critical volumes of aqueous homogeneous solutions of uranium in defining the 2200-m/sec neutron parameters for ENDF/B has been examined. The parameters for ${ }^{23}{ }^{3} U$ and ${ }^{235} \mathrm{U}$ are constrained by relating $(\bar{n}-1) \bar{\sigma}_{a x}$ to the constant $K$ obtained from the analys is of the critical systems. $K$ is directly proportional to the hydrogen capture cross section at $2200 \mathrm{~m} / \mathrm{sec}$. This paper suggests that the capture cross section of hydrogen be removed from $K$ and that a new constant $K / \sigma_{a H}$ be defined by the critical systems. This new constant is the hydrogen to uranium ratio for an infinite critical system populated with neutrons having a Maxwellian energy distribution.

b. Analysis of Neutron Scattering and Gamma-Ray Production Integral Experiments on Nitrogen for Neutron Energies from 1 to $15 \mathrm{MeV}$ ** (S. N. Cramer and E. M. ObTow)

Two integral experiments of neutron scattering and gamma-ray production from nitrogen samples performed at Intelcom Radiation Technology and Oak Ridge National Laboratory were analyzed with Monte Carlo calculations. The experimental results include angular-dependent NE-213 detector count rates for both scattered neutrons and gamma-ray production from a spherical dewar of 1 iquid nitrogen pulsed with a neutron beam with energies from 1 to $20 \mathrm{MeV}$. Additional results were reported in the ORNL experiments for unfolded scattered neutron and gamma-ray production spectra as a function of detector angle in broad incident neutron energy bins. Multigroup Monte Carlo calculations were made to analyze all the reported results. Conclusions were drawn about the status of the ENDF/B-IV nitrogen cross-section data file from the comparison of calculated and experimental results.

* Abstract of ORNL-TM-4550 (January 19/5); paper submitted for publication in Nuclear Science and Engineering.

** Abstract of ORNL/TM-5220. 
c. Analysis of Neutron Scattering and Gamma-Ray Production Integral Experiments on Carbon for Neutron Energies from 1 to $14 \mathrm{MeV}$ * (S. N. Cramer and E. M. Oblow)

The results of two integral experiments on carbon, performed at ORNL and IRT, were compared with Monte Carlo calculations to test evaluated carbon neutron and gamma-ray production data sets. In both experiments NE-213 detectors were used to measure the angular dependence of neutron scattering and gamma-ray production from thick ( $1 \mathrm{mfp}$ ) carbon sample in the energy range from 0.5 to $20 \mathrm{MeV}$. Additional measurements from the ORNL experiment also provided angular-dependent, energy distributions of the scattered neutrons. Multigroup Monte Carlo calculations , odeling the two experimental arrangements were made to compare with the measured data. Both ENDF/B-III and ENDF/B-IV carbon data were used in the computations. The results indicate that such experiments are adequate for testing processed neutron scattering and gamma-ray production data (both integral and double differential) to within 10-20\% over a wide range of incident neutron energies ( 1 to $15 \mathrm{MeV}$ ). Also, on the whole, calculations with the carbon ENDF/B-IV data compared favorably with the measured results over the energy range tested. The only notable exceptions were the disagreements in the neutron result comparisons above $9 \mathrm{MeV}$ which were attributed for the most part to errors in the evaluated $c\left(n, n^{\prime}\right) 3 \alpha$ and elastic angular distribution cross sections in this range.

d. Comparison of Measurements and Calculations for ORNL Integral Neutron Scattering Experiment for Iron** (S. N. Cramer and E: M. Oblow)

Calculations of an integral neutron scattering experiment on an iron sample have been performed using the ENDF/B-IV data set MAT 1192 (DNA MAT 4192). The neutron source incident on the sample ranged from $20 \mathrm{MeV}$ to $1 \mathrm{MeV}$. Comparisons between experimental and calculated results are given as neutron count rates and neutron secondary energy spectra.

e. Experiment on Secondary Gamma-Ray Production Cross Sections Averaged Over a Fast-Neutron Spectrum for Each of 13 Different Elements Plus a Stainless Steelt (K. E. Maerkér)

The experimental and calculational details for a CSEWG integral data testing shielding experiment are presented. This particular experiment measured the secondary gamma-ray production cross sections averaged over a fast-neutron spectrum for iron, oxygen, sodium, aluminum, copper, titanium, calcium, potassium, silicon, nickel, zinc, barium, sulfur, and a type 321 stainless steel. The gamma-ray production cross sections were binned into $\sim 0.5-\mathrm{MeV}$ wide gamma-ray energy intervals.

* Abstract of paper submitted for publication in Nucl. Sci. Eng.

** Abstract of paper to be presented at ANS Meeting, June 1976; Toronto, Canada.

+ Abstract of ORNL-TM-5204, ENDF-228 (January 1976). 
f. Neutron Total Cross Section Checks for Iron, Chromium, Nickel, Stainless Steel, Sodium and Carbon* (R. E. Maerker and F. J. Muckenthaler)

Utilizing detectors in good geometry at the Tower Shielding Facility behind various thicknesses of iron, chromium, and nickel provided experimental data for checking neutron total cross sections of these elements. In addition, data behind a type $304 \mathrm{~L}$ stainless steel and carbon were also obtained, as were some data behind sodium. These data were then compared with data from the total cross section files of both versions III and IV of ENDF/B. Results of these comparisons indicate version IV to be generally superior to version III, but further improvement in version IV is possible for all elements tested. In particular, minima in the total cross sections for both chromium and nickel are apparently still poorly represented in version IV for all energies above $10 \mathrm{keV}$.

\section{Sensitivity Studies}

a. The Frequency of Occurrence of Various Nuclear Reactions When Fast Neutrons ( $50 \mathrm{MeV}$ ) Pass Through Tissue-Equivalent Material** (R. G. Alsmiller, Jr. and J. Barish)

Calculated results are presented for the frequency with which various partial nuclear-reaction cross sections are utilized when fast neutrons ( $\approx 50 \mathrm{MeV}$ ) are transported through a tissue-equivalent phantom to obtain an indication of which cross sections are of most importance for radiotherapy applications and are therefore in need of experimental verification.

b. Survey of Shielding Sensitivity Analysis Development and Applications Program at ORNL广 (E. M. Oblow)

The cross-section sensitivity analysis program at ORNL is reviewed with emphasis on present computer code capabilities and past successful applications in the radiation shielding area. The FORSS sensitivity code system is discussed in regard to objectives, methodology, and code specifications. Examples of past shielding applications of FORSS emphasize the success of fine energy grid sensitivity studies and group structure selection, the use of evaluated error files in problem uncertainty estimation, two-dimensional shield sensitivity analysis and integral experiment design for fast reactors, data studies for the LMFBR program

* Abstract of ORNL-5013 (January 1976).

** Abstract of ORNL-TM-4970.

† Abstract of ORNL-TM-5176; paper presented at the Specialists' Meeting on Sensitivity Studies and Shielding Benchmarks, IECD - Paris, France, October 7-10, 1975. 
related to sodium and iron evaluations, and iron data problems in CTR shield design. Conclusions are drawn about the adquacy of present ENDF/B data files for sodium and iron and the general applicability of sensitivity studies in future shield design and analysis.

c. Preliminary Cross-Section Sensitivity Analys is for an Air-OverGround Environment* (J. V. Pace, III and D. E. Bartine)

Two-dimensional sensitivity calculations have been made for an air-over-ground geometry to determine the effect of air and ground crosssection perturbations on the total neutron and gamma-ray dose near the air/ground interface and 415 meters above the ground. EN F/B 22 neutron and 18 gamma group Version IV cross sections were used in all computations.

d. Cross-Section Sensitivity of the D-T Fusion Probability and the D-T and T-T Reaction Rates** (R. T. Santoro and J. Barish)

The cross-section sensitivity of the fusion probability has been calculated for various conditions of incident deuteron energy and plasma electron temperature. The fusion probability is most sensitive to the D-T cross section at the higher energies ( $Z 50 \mathrm{keV})$, and, based on the reported errors in the cross section, the errors in the calculated fusion probabilities should be $\lesssim 10 \%$.

The cross-section sensitivities of the $D-T$ reaction rate in a $D-T$ plasma and the T-T reaction rate in a tritium plasma have also been calculated for various assumed values of the plasma ion temperature.

e. Uncertainties in Calculated Heating and Radiation Damage in the Toroidal Field Coil of a Tokamak Experimental Power Reactor due to Neutron Cross-Section Errorst (Alsmiller, Barish and Weisbin)

Calculated results are presented of the uncertainties in the neutron scalar flux, the energy deposition per unit volume, and the displacements per atom in the toroidal field coil of a tokamak experimental. power reactor due to neutron cross-section errors iniron and carbon which are major constituents of the blanket-shield-coil configuration considered. The calculations were carried out using perturbation theory to obtain sensitivity profiles for the various cross sections of interest, and these profiles were then combined with cross-section error estimates, including correlations, to obtain the uncertainties.

* Abstract of paper presented at ANS Meeting, San Francisco, November $16-21,1975$.

$\star \star$ Abstract of ORNL-TM-4933.

+ Abstract of ORNL/TM-5198 (March 1976); paper submitted to Nucl. Sci. Eng. 
Each of the three responses - the neutron scalar flux, the energy deposition per unit volume, and the displacements per atom - is found to be very sensitive to the cross sections in the energy group which contains the source ( $\sim 14 \mathrm{MeV}$ since a D-T source is assumed), and each of the responses is found to have a relative standard deviation of approximately $100 \%$ due to neutron cross-section errors in iron.

f. Neutronics Calculations for the Tokamak Experimental Power Reactor Reference Design* (R. T. Santoro)

The results of initial one-dimensional calculations evaluating the nuclear performance of the Tokamak Experimental Power Reactor are presented. Estimates of the tritium breeding, nuclear heating, and radiation damage are given for an assumed neutron wall loading of $0.168 \mathrm{MW} / \mathrm{m}^{2}$ (100 MW).

g. Neutronic Scoping Studies for Tokamak Experimental Power Reactor** (Santuro, E. S. Bettis, + D. G. McATees, 中 H. L. Wátts ànd M. L. Williams)

One-dimensional neutron and photon radiation transport methods have been used to investigate candidate blanket configurations and compositions for use in the Tokamak Experimental Power Reactor. Seven blanket designs are compared in terms of energy recovery, radiation attenuation, potential radiation damage, and, where applicable, tritium breeding.

h. The Calculated Performance of Various Structural Materials in Fusion-Reactor Blankets T (Williams, Santoro and Gabriel)

The calculated nuclear performances of niobium, SS-304, and nimonic- 105 as structural materials in a conceptual $\bar{D}-\bar{T}$ fusion-reactor blanket model are compared. For each structural material, the tritium breeding ratio, the energy-deposition rate, the operating dose, the time dependence of the neutron-induced activity, the time dependence of the dose from the activation products, the time dependence of the nuclear afterheat, and the atomic displacement rate are calculated. Emphasis is placed on the nuclear response in the first structural wall to the selected structural material for an assumed neutron wall loading of $1 \mathrm{MW} / \mathrm{m}^{2}$. Taking into account all of the nuclear responses, SS-304 appears to be a reasonable choice as the structural material for fusion-reactor applications.

* Abstract of ORNL-TM-5033.

** Abstract of ORNL/TM-5035 (February 1976).

+ Consultant to Thermonuclear Division

キ Exxon Nuclear Company, Inc.

I Abstract of ORNL-TM-5036 (December 1975); paper submitted to Nuclear Technology. 
5. Multigroup Libraries

After all the nuclear data have been evaluated in the form of microscopic cross sections or data as a function of energy in a library such as ENDF/B, they can then be used directly for applied calculations. However, in most instances, in particular in applications involving neutron transport, the data must be processed in the form of multigroup cross sections. Until a few years ago most users generated for themselves the multigroup data from the evaluated data files. With the increase in size of the evaluated data files, as they became more detailed and complete, the cost of generating the multigroup data sets and maintaining the processing codes has soared and become prohibitive for many users. As a result, a demand has arisen for processed nuclear data. When processing the evaluations, the group structure must be tailored to the particular application. The Radiation Shielding Information Center has traditionally provided these processed data sets to users and during 1975 several multigroup libraries were generated from ENDF/B-IV for various applications.

We report below on a few of these data sets generated at ORNL and available from RSIC.

a. A 218-Group Master Neutron Cross-Section Library for Criticality Safety Studies* (W. E. Ford, III, C. C. Webster and R. M. Westfal1)

$A \cdot P_{3}, 218$ neutron group master cross-section library has been generated from the latest ENDF/B data for 65 nuclides of primary interest in criticality safety calculations. Procedures used to generate the cross sections and the organization of the master cross-section library are described.

b. Coupled 100-Group Neutron and 21-Group Gamma-Ray Cross Sections for EPR Calculations** (D. M. Plaster, R. T. Santoro and W. E. Ford, III)

Coupled 100-group neutron and 21 -group gamma-ray $800^{\circ} \mathrm{K}$ cross sections have been generated using the latest ENDF/B cross-section data for $\mathrm{H}$, He, ${ }^{6} \mathrm{Li},{ }^{7} \mathrm{Li}, \mathrm{Be},{ }^{10} \mathrm{~B},{ }^{11} \mathrm{~B}, \mathrm{C}, 0, \mathrm{~F}, \mathrm{Al}, \mathrm{Ti}, \mathrm{V}, \mathrm{Cr},{ }^{55} \mathrm{Mn}, \mathrm{Fe},{ }^{59} \mathrm{Co}$, $\mathrm{Ni}, \mathrm{Cu}, \mathrm{Nb}$, and $\mathrm{Pb}$. The procedures used to generate these cross sections and the organization of the cross-section tape in ANISN format are discussed.

* Abstract of ORNL/CSD/TM report (to be published).

** Abstract of ORNL-TM-4872. 
Modification Number One to the Coupled 100n-21y Cross Section Library for EPR Calculations* (Ford, Santoro, Roussin and Plaster)

The EPR ANISN-formatted 100-group neutron and 21-group gamma-ray cross section library has been modified by the addition of data for 20 materials, by the addition of reaction cross sections for calculating tritium and helium production, and by the addition of kerma factors. The EPR 100-group master cross section library has been modified by the addition of data for 19 materials. Procedures used to generate these cross sections and the organization of the libraries are described.

\section{c. Data Testing of the 126/36 Neutron-Gamma ENDF/B-IV Coupled Library for LMFBR Core and Shield Analysis** (White, Wright, Willianls arid Weistin)}

The Physics Branch of the ERDA Division of Reactor Development and Demonstration has sponsored the development of a 126/36 neutron/gamma coupled library derived from ENDF/B-IV data. The detailed specifications (e.g., selection of the tailored group structure) and processing methods have already been documented. ${ }^{1}$ The purpose of this paper is to describe the testing program and results obtained to date in an effort to gain confidence in the library performance for a variety of important LMFBR core and shield applications.

d. The CTR Processed Multigroup Cross Section Library for Neutronics Studiest (Roussin, Weisbin, White, Greene, R. Q. Wright and J. B. Wright)

The program for the development, generation, validation and distribution of a general purpose, processed multigroup cross section library (PMCSL) for use in CTR neutronics studies is described. The Radiation Shielding Information Center (RSIC) coordinated an effort to establish the CTR-PMCSL based on input requirements specified by ERDA-DCTR contractors. By collaborating with the ORNL Neutron Physics Division Shielding Analysis and Reactor Physics Group (which had a similar mission for the ERDA-DRDD community) it was possible to define a single 171 neutron, 36 gamma-ray group cross-section library useful for both CTR and LMFBR neutronics analysis. The master library was generated using the MINX

* Abstract of ORNL/TM-5249

** Abstract of paper to be presented at ANS Meeting, June 1976, Toronto.

+ Abstract of ORNL/RSIC-37; paper to be presented at ANS Meeting, Jurle 1976, Toronto.

1 C. R. Weisbin, R. W. Roussin, J. E. White and R. Q. Wright, "Specification for Pseudo-Composition-Independent Fine-Group and CompositionDependent Fine- and Broad-Group LMFBR Neutron-Gamma Libraries at ORNL," ORNL-TM-5142 (ENDF-224) (December 1975). 
neutron processor and the gamma-ray processor from the AMPX system. Results of initial testing of the library at ORNL are described. Preliminary release of the library is being made to a group of ERDA contractors in AMPX and CCCC interface forms, along with appropriate retrieval and manipulation programs. Problem-independent and problemdependent collapsed versions will also be provided. The release is intended to stimulate implementation and testing at several installations for the purpose of improving the quality of the libraries which will ultimately receive general distribution.

e. Radiation-Damage Calculations: Primary Recoil Spectra, Displacement Rates, and Gas-Production Rates* (T. A. Gabriel, J. D. Amburgey and N. M. Greene).

A heavy charged-particle recoil data base [primary knock-on atom (PKA) spectra] and an analysis program have been created to assist experimentalists in studying, evaluating, and correlating radiation-damage effects in different neutron environments. Since experimentally obtained controlled-thermonuclear-reactor-type neutron spectra are not presently available, the data base can be extremely useful in relating currently obtainable radiation damage to that which is anticipated in future fusion devices. However, the usefulness of the data base is not restricted to just CTR needs. Most of the elements of interest to the radiation-damage community and all neutron reactions of any significance for these elements have been processed, using available ENDF/B-IV cross-section data, and are included in the data base. Calculated data such as primary recoil spectra, displacement rates, and gas-production rates, obtained with the data base, for different radiation environments are presented and compared with previous calculations.

* Abstract of ORNL/TM-5160 (March 1976). 


\section{COMPILATION AND NUCLEAR DATA PROJECT}

1. Compilation of Phenomenological Optical-Model Parameters 1954-1975* (C. M. Perey and F. G. Perey)

Presented here is a compilation, with bibliography, of opticalmodel parameters determined by fitting elastic-scattering angular distributions for various incident particles including heavy ions. It includes parameters from previous compilations back to 1954 and from an extensive literature search in the leading journals and publications in nuclear physics up to June 1975 inclusively.

2. Nuclear Data Project Activities 1975 (R. L. Aublc, **

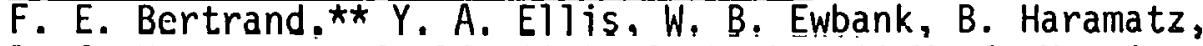
D. J. Horen, H H. J. Dim, $* \star$ D. C. Kocher, ** M. J. Martin, F. K. McGowan, ** and M. R. Schmorak)

Ihis year has seen the culmination of Nuclear Data Project' (NDP) efforts in several areas. The Evaluated Nuclear Structure Data File (ENSDF) has been developed to where it can serve as the focus for NDP evaluation activities. Standard ENSDF data sets are being used routinely to produce computer-printed Nuclear Data Sheets as well as drawings. Additional output formats are being developed to meet needs of other users. The Project's file of keyword-indexed references to nuclear structure literature has also been exploited in new ways during the year: a volume of cumulated references to the experimental nuclear structure literature (1969-1974) was issued as a Supplement to Volume 16 of Nuclear Data Sheets; the contents of the cumulated volume were also placed on the RECON system for direct interactive search by any qualified user.

The standard formats developed by the Nuclear Data Project for the representation of nuclear structure data are gaining wider understanding and acceptance as the existence of ENSUF becomes known. Tape copies of data sets containing adopted levels and decay schemes have been sent on request to several laboratories in the U.S., Europe, and the Soviet Union. The data center at LINP (Leningrad Institute of Nuclear Physics) has also used the standard formats to describe results of nuclear physics experiments.

\section{a. Evaluated Nuclear Structure Data File (ENSDF)}

The data file was designed to organize nuclear structure information in standard format into convenient blocks and to provide tools for. storing and retrieving the information as needed. Information in ENSDF is organized into "data sets" of three general types: decay schemes of

* Abstract of paper submitted for publication in Atomic Data and Nuclear Data Tables.

** Part-time assignment to Nuclear Data Project.

+ Director, Nuclear Data Project until November 1975. 
radioactive nuclei, level structure from nuclear reactions, and adopted level properties. The file is maintained on disk at the ORNL computer center. As of March 1974 the file contained

\author{
1230 decay schemes \\ 1800 reaction data sets \\ 1540 adopted levels data sets.
}

Selection of particular data from the ENSDF is accomplished by computer scanning of a descriptive keyword string.

\title{
b. Nuclear Data Sheets from ENSDF
}

For several years, the drawings in Nuclear Data Sheets have been prepared from. standard ENSDF data sets. During 1975, the Nuclear Data Project also began preparation of the printed Data Sheets from the data file. A standard page layout for presentation of tabular data was first used for $A=75$. Addition of a text editor to control the special ORNL 160-character print train now produces acceptable computer-composed pages for photoreproduction.

The NDS listing program has also been used to prepare special responses to requests for nuclear structure information, e.g., a table of all nuclear levels with known lifetimes, an ordered list of $\gamma$-rays observed in decay of transactinide nuclei, tabulation of levels and $\gamma$-rays observed in $(H I, x n Y)$ reactions.

\section{c. Decay Radiations from ENSDF}

One additional output format for information from ENSDF has been tested. The MEDLIST program, which calculates absolute intensities for both atomic and nuclear radiations following radioactive decay, normally prepares a tabular format for photoreproudction (e.g., ORNL-5114). The program was extended in 1975 to also provide a file of these radiations in a card-image format, easily readable by FORTRAN programs. Many conventions of the ENDF/B-IV tapes were adopted to facilitate inclusion of ENSDF data in some future version of ENDF. Radiations from 191 radioactive nuclei were written onto a tape, which has been sent to several users for testing. A copy is also available on data cell for testing by ORNL users of radioactivity data.

Discussions with MIRD representatives and members of the Information Center for Internal Exposure at ORNL have produced tentative agreement to use ENSDF data in future calculations for medical dosimetry and for radiation protection standards.

\section{d. Nuclear Structure References}

In support of its data evaluation program, the Nuclear Data Project searches the world's scientific literature for reports of 
nuclear structure results. Each year approximately 3000 published articles and 2000 preliminary reports are tagged according to data content and added to the indexed nuclear structure reference file. The master file now includes some 55,000 entries on magnetic tape at ORNL.

The cumulated file of Nuclear Structure References, 1969-1974, has been prepared for inclusion among the reference files available from the RECON network. The file can be searched for keyword descriptions and references on nuclear structure topics from any of 27 directly connected RECON sites within the USA. Since the introduction in mid1975 of a dial-up version of RECON, any ERDA-approved user can search the files from any telephone. The RECON version of the NDP reference tiles does not take full advantage of the keywurd structure, but it does provide an interactive search capability which can include combinations of key terms (isotope, reaction, etc.). The RECON file can also be used to search on author or publication year, and can provide printed references if needed. 
INTERNAL DISTRIBUTION

$\begin{aligned} & \text { 1-3. L. S. Abbott } \\ & \text { 4. R. G. Alsmiller, Jr. } \\ & \text { 5. G. T. Chapman } \\ & \text { 6. } \text { C. E. Clifford } \\ & \text { 7. J. W. T. Dabbs } \\ & \text { 8. G. de Saussure } \\ & \text { 9. } \text { J. K. Dickens } \\ & \text { 10. W. B. Ewbank } \\ & \text { 11. } \text { J. L. Fowler } \\ & \text { 12. C. Y. Fu } \\ & \text { 13. J. C. Gentry } \\ & \text { 14. R. Gwin } \\ & \text { 15. J. A. Harvey } \\ & \text { 16. D. J. Horen } \\ & \text { 17. C. H. Johnson } \\ & \text { 18. W. E. Kinney } \\ & \text { 19. D. C. Larson } \\ & \text { 20. R. L. Macklin } \\ & \text { 21. F. C. Maienschein } \\ & \text { 22. B. F. Maskewitz } \\ & \text { 23. G. L. Morgan } \\ & \text { 24. } \text { F. R. Mynatt }\end{aligned}$

25. E. Newman

26. E. M. Oblow

27. R. W. Peelle

28-32. F. G. Perey

33. S. Raman

34. R. W. Rouss in

35. G. G. Slaughter

36. D. Steiner

37. P. H. Stelson

38. C. R. Weisbin

39. L. W. Weston

40. A. Zucker

41. P. F. Fox (Consultant)

42. W. W. Havens, Jr. (Consul tant)

43. A. F. Henry (Consultant)

44. R. E. Uhrig (Consultant)

45-46. Central Research Library

47. ORNL Y-12 Technical Library, Document Reference Section

48. ORNL Patent Office

49. Laboratory Records Department

50. Laboratory Records ORNL RC

\section{EXTERNAL DISTRIBUTION}

51. John D. Anderson, Physics Dept., E Division, Lawrence Livermore Laboratory, P. 0. Box 808, Livermore, CA 94550

52. David Auton, Defense Nuclear Agency, Washington, DC 20305

53. Robert C. Biock, Nuclear Eng. \& Science Dept., Rensselaer Polytechnic Inst., Troy, NY 12181

54. Charles D. Bowman, Radiation Division, National Bureau of Standards, Washington, DC 20234

55. J. C. Browne, Lawrence Livermore Laboratory, P. 0. Box 808, Livermore, CA 94550

56. Randa11 S. Caswell, Deputy Director, Center for Radiation Research, National Bureau of Standards, Washington, DC 20234

57. Robert E. Chrien, Department of Physics, Brookhaven National Laboratory, Upton, NY 11973

58. D. Eccleshall, U.S. Army Ballistic Research Laboratories, Aberdeen Proving Ground, MD 21005

59. A. Elwyn, Argonne National Laboratory, Argonne, IL 60439

60. D. Gardner, Radiochemistry Division, Lawrence Livermore Laboratory, Livermore, CA 94550

61. G. T. Garvey, Physics Section, National Science Foundation, Washington, DC 20550

62. Herbert Goldstein, Div. of Nuclear Science \& Engineering, Room 242, S. W. Mudd Building, Columbia University, New York, NY 10027 
63. B. G. Harvey, Deputy Division Leader, Nuclear Chemistry Division, Lawrence Berkeley Laboratory, Berkeley, CA 94720

64. R. L. Heath, Research \& Engineering, Aerojet Nuclear Company, Idaho Falls, ID 83401

65. Philip B. Hemmig, Chief, Physics Branch, Div. of Reactor Dev. and Demonstration, U.S. Energy Research \& Dev. Admin., Washington, DC 20545

66. Robert W. Hockenbury, Nuclear Engineering \& Science Department, Rensselaer Polytechnic Institute, Troy, NY 13181

67. Harold E. Jackson, Physics Division, Argonne National Laboratory, Argonne, IL 60439

68. G. F. Knol1, University of Michigan, Ann Arbor, MI 48104

69. R. 0. Lane, Accelerator Laboratory, Department of Physics, Ohio University, Athens, $\mathrm{OH} 45701$

70. M. T. Me[1listrem, Department of Physics \& Astronomy, University of Kentucky, Lexington, $\mathrm{KY} 40506$

71. Michael S. Moore, P. Division, Los Alamos Scientific Laboratory, Los Alạmos, NM 87545

72. Henry T. Motz, P Division Leader, Los Alamos Scientific Laboratory, Los Alamos, NM 87545

73. Henry W. Newson, Department of Physics, Duke University, Durham, NC 27706

74. Sol Pearlstein, Director, NNCSC, Brookhaven National Laboratory, Upton, NY 11973

75. George L. Rogosa, Assistant Director for Nuclear Sciences, Division of Physical Research, U.S. Energy Research \& Dev. Admin., Washington, DC 20545

76. H. L. Schultz, Physics Department, Yale University, New Haven, CT 06520

77. Alan B. Smith, Applied Physics Division, Argonne National Laboratory, Argonne, IL 60439

78. Bruce Twining, Systems \& Applications Studies Branch, Div. of Controlled Thermonuclear Res., U.S. Energy Research \& Dev. Admin., Washington, DC 20545

79. Stanley Whetstone, Nuclear Science, Div. of Physical Research, U.S. Energy \& Dev. Admin., Washington, DC 20545

80. P. G. Young, Assistant Group Leader, T-2 Nuclear Data, Los Alamos Scientific Laboratory, Los Alamos, NM 87545

81. U.S. ERDA Oak Ridge Operations, Research and Technical Support Division, P. O. Box E, Oak Ridge, TN 37830: Directór

82-108. Technical Information Center (TIC) 